\title{
Nonlinear Aeroelastic Behavior of an Airfoil with Free-play in Transonic Flow
}

\author{
Shun $\mathrm{He}^{\mathrm{a}}$, Shijun $\mathrm{Guo}^{\mathrm{a}, *}$, Wenhao $\mathrm{Li}^{\mathrm{a}}$, Daqing Yang ${ }^{\mathrm{a}}$, Yingsong $\mathrm{Gu}^{\mathrm{b}}$, \\ Zhichun Yang ${ }^{\mathrm{b}}$ \\ ${ }^{a}$ Centre for Aeronautics, Cranfield University, Cranfield, Bedfordshire, UK, MK43 $0 A L$ \\ ${ }^{b}$ School of Aeronautics, Northwestern Polytechnical University, Xi'an, People's Republic \\ of China, 710072
}

\begin{abstract}
An investigation has been made into the nonlinear aeroelastic behavior of an airfoil system with free-play nonlinear stiffness in transonic flow. Computational Fluid Dynamics (CFD) and Reduced Order Model (ROM) based on Euler and Navier-Stokes equations are implemented to calculate unsteady aerodynamic forces. Results show that the nonlinear aeroelastic system experiences various bifurcations with increasing Mach number. Regular subcritical bifurcations are observed in low Mach number region. Subsequently, complex Limit Cycle Oscillations (LCOs) and even non-periodic motions appear at specific airspeed regions. When the Mach number is increased above the freeze Mach number, regular subcritical bifurcations occur again. Comparisons with inviscid solutions are used to identify and elaborate the effect of viscosity with the help of aeroelastic analysis techniques, including root locus, single degree of freedom flutter and aerodynamic influence coefficient

${ }^{*}$ Corresponding author.

Email addresses: shun.he@cranfield.ac.uk / nwpuheshun@gmail.com (Shun He), s.guo@cranfield.ac.uk (Shijun Guo)
\end{abstract}


(AIC). For low Mach numbers in the transonic regime, the viscosity has little effect on the linear flutter characteristic because of limited influence on AIC, but a remarkable impact on the nonlinear dynamic behavior due to the sensitivity of the nonlinear structure. As the Mach number increases, the viscosity becomes significantly important due to the existence of shock-boundary layer interaction. It affects the unstable mechanism of linear flutter, impacts the aerodynamic center and hence the snap-through phenomenon, influences the AIC and consequently the nonlinear aeroelastic response. When the Mach number is increased further, the shock wave dominates the air flow and the viscosity is of minor importance.

Keywords: Free-play, Nonlinear aeroelastic response, Viscous flow, Transonic flutter, Chaos

\section{Introduction}

The aroelastic behavior of airfoil systems in the transonic regime has attracted researchers for decades. A common observation in the transonic flow region is that the critical flutter speed is significantly reduced, a phenomenon known as "transonic dip" [1]. Another feature is that the response typically evolves into an LCO rather than a divergent response. The LCOs can be induced by nonlinear aerodynamic effects such as shock wave in transonic flow [2]. It is known that aerodynamic instabilities can occur in the boundary layer of an airfoil that lead to flow separation, and the flow separation and the shock-boundary layer interaction may enhance the nonlinear effect of aerodynamics. In terms of the structure model, a conventional aeroelastic analysis is usually based on the assumption of linear structure. In reality, 
however, the structural system can be subject to nonlinear stiffness such as free-play, hysteresis or cubic nonlinearity, which affects its aeroelastic behavior [3]. So investigation of the dynamic response of aeroelastic system with nonlinear stiffness in transonic flow is of particular interest.

As a high-fidelity technique to capture shock wave and flow separation, CFD method has been widely applied to carry out the aeroelastic response of the nonlinear structural model particularly in transonic air flow. Kousen and Bendiksen [4] investigated the LCO of an aeroelastic airfoil with a free-play nonlinearity in the pitching degree of freedom (DOF). In their study, the bifurcation diagram was obtained by using time marching approach based on Euler equations. The inviscid transonic aeroelastic behavior of an airfoil with free-play nonlinearities in both pitching and plunging DOFs was analyzed by Kim and Lee [5], and LCOs and chaotic motions were observed in specific ranges of Mach numbers. Based on Euler equations, Yang et al. [6] investigated the LCO behaviors of an aeroelastic airfoil with free-play at different Mach numbers. An interesting chimney region on the flutter boundary was presented and well explained. The aeroelastic response of a nonlinear panel in transonic and low supersonic air flow was studied by Shishaeva et al. [7]; substantial various nonlinear phenomenon including bifurcation, coexistence of different limit cycles and non-periodic oscillations were observed in accelerating or decelerating air flow conditions. A three-DOF aeroelastic airfoil with a free-play in the control surface was investigated in transonic air flow by means of Euler-based CFD, and higher-order spectra techniques [8] and HilbertHuang Transform techniques [9] were applied to understand the features and physical reasons of observed transition between different type 
of nonlinear aeroelastic responses.

The ROM method is applied in the problem studied here to reduce the computational cost in CFD simulation. Using proper orthogonal decomposition method and Euler solution, a three-DOF aeroelastic wing model with free-play in control surface was implemented to study the transonic LCO behavior by Dowell et al. [10]. The so-called chimney phenomenon was observed in the flutter boundary. The LCOs of an aeroelastic airfoil with structural nonlinearity in subsonic and transonic air flow were also obtained by Munteanu et al. [11] using the ROM technique for a viscous solution. This study indicates that the ROM approach can be used to estimate the LCOs accurately and efficiently. An airfoil with piecewise nonlinearity was investigated by Jones et al. [12] in both low speed incompressible flow and transonic air flow based on the Euler equations, and the LCOs were rapidly identified with good accuracy. It should be specially mentioned that a series of interesting researches based on aerodynamic ROM technique were carried out by Gao et al. $[13,14,15]$, to explore the mechanism of frequency lock-in phenomenon in transonic flutter and buffeting.

Previous work on the nonlinear dynamic behavior of an aeroelastic airfoil with free-play in inviscid transonic flow has been carried out by the authors [16]. It revealed the significant features of the responses over different flow speed regions but at fixed transonic Mach number (Mach 0.87), which can be summarized as:

- The aeroelastic responses with the amplitude near the free-play are dominated by the single degree of freedom flutter mechanism, and snapthrough phenomenon is observed when the airspeed is low. 
- The route to chaos is via period-doubling, which is primarily caused by the free-play nonlinearity.

- The aeroelastic response is dominated by aerodynamic nonlinearity when air velocity is around the linear flutter speed.

Here, we extend our previous study [16] to consider the dependence of the nonlinear dynamic behavior of the aeroelastic system to Mach number, which is rarely reported in the existing literature. So the first aim of the present study is to exhibit the nonlinear dynamic evolution of the aeroelastic system considering Mach number as a control parameter.

As mentioned above, most of the existing research activities on transonic nonlinear flutter were carried out by using inviscid (Euler) solution methods. Inviscid flow is normally assumed to simplify the aerodynamic solution, and there are many practical aerodynamic applications where this assumption is valid [17]. However, the fact is that the real air flow is always viscous in nature. In addition, the numerical analysis on an aeroelastic airfoil of NACA 0012 [18] and AGARD 445.6 wing [19] has highlighted that the aerodynamic viscosity has a significant impact on the transonic flutter characteristics. But it remains unclear whether the assumption of inviscid flow affects the nonlinear response of an aeroelastic system in transonic regime. Thus, the second aim of the present study is to determine the effect of the aerodynamic viscosity on the transonic linear and nonlinear aeroelastic behavior, and to assess the suitability of inviscid flow to model the transonic unsteady aerodynamics for aeroelastic problems.

Based on Euler equations and Navier-Stokes equations, the nonlinear dynamic behaviors of an aeroelastic airfoil with free-play over a wide range of 
transonic Mach numbers are studied. With increasing Mach number, complex LCOs and non-periodic motions occur over specific airspeed ranges. Regular subcritical bifurcations are observed at low Mach numbers. As the Mach number is increased higher than the freeze Mach number of the airfoil, regular subcritical bifurcations happen again. By comparing the aeroelastic behavior of inviscid and viscous solution, the effect of viscosity on aeroelastic behavior is identified. It is found that the viscosity plays different roles at different Mach number ranges, and the suitability of inviscid solution depends on the Mach number as well as the type of the aeroelastic problem.

This paper is organized as follows. In section 2 , the governing equations of motion and technical methods are formulated. Mesh independence study, time step convergence study and verification of numerical approaches are performed in section 3. Section 4 and 5 present linear flutter solution and nonlinear dynamic behaviors respectively, and the effect of viscosity is discussed as well. Finally, conclusions are drawn in section 6 .

\section{Technical analysis}

\subsection{Governing equations for an aeroelastic airfoil}

Figure 1 shows a sketch of an aeroelastic airfoil with plunging $(h)$ and pitching $(\alpha)$ DOFs. The elastic axis of the airfoil (E point) is located at a distance of $a b$ rear of the mid-chord point, the gravity center (G point) is located at $x_{a} b$ behind the elastic axis, and the aerodynamic center of the airfoil is located at $x_{a c} b$ after the mid-chord point, where $b$ is the half-chord length. The mass per unit span is $m$, the first moment of inertia about the elastic axis is $S_{\alpha}=m x_{\alpha} b$, and the moment of inertia about the elastic axis 


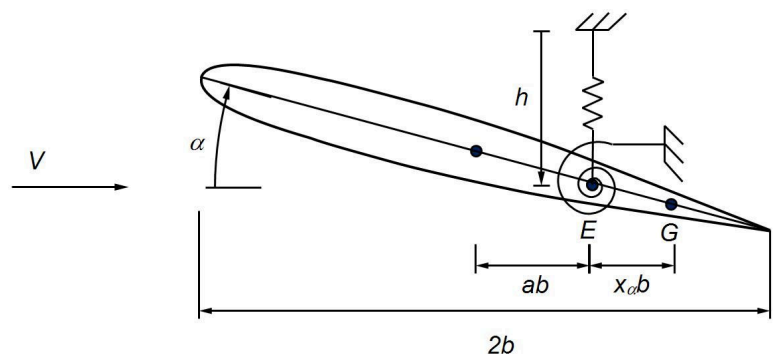

Figure 1: An aeroelastic airfoil in transonic air flow.

is $I_{\alpha}=m r_{\alpha}^{2} b^{2}$. The bending stiffness and torsion stiffness are modelled by springs attached to the elastic axis. A linear spring is considered in plunging DOF, and the plunging stiffness coefficient is $K_{h}=m \omega_{h}^{2}$. While a free-play nonlinearity is assumed in the pitching DOF, and the nonlinear structural restoring moment can be described as

$$
M(\alpha)=\left\{\begin{array}{lc}
K_{\alpha}(\alpha-\delta) & \alpha \geq \delta \\
0 & -\delta<\alpha<\delta \\
K_{\alpha}(\alpha+\delta) & \alpha \leq-\delta
\end{array}\right.
$$

where $\delta$ denotes the measurement of free-play, $K_{\alpha}=I_{\alpha} \omega_{\alpha}^{2}$ is the torsion stiffness coefficient. To express the equations in matrix form in the following parts of this section, Eq. (1) can also be rewritten as

$$
M(\alpha)=K_{\alpha} \alpha+K_{\alpha}\left\{\begin{array}{cc}
-\delta & \alpha \geq \delta \\
-\alpha & -\delta<\alpha<\delta \\
\delta & \alpha \leq-\delta
\end{array}=K_{\alpha} \alpha+K_{\alpha} f_{\text {non }}\right.
$$

Note that when $\delta=0, f_{\text {non }}=0$ and the airfoil is reduced to a linear structural model. Viscous damping is considered with the damping coefficients of $D_{h}=$ $2 \zeta_{h} m$ in plunging and $D_{\alpha}=2 \zeta_{\alpha} I_{\alpha}$ in pitching. 
The governing equations of motion for the linear structure were derived from the Lagrange equations according to Dowell et al. [20]. The nonlinear structural restoring moment from the spring with free-play in pitching DOF is considered in the present study. The nonlinear governing equations can be expressed as

$$
\left\{\begin{array}{l}
m \ddot{h}+S_{\alpha} \ddot{\alpha}+D_{h} \dot{h}+K_{h} h=-L \\
S_{\alpha} \ddot{h}+I_{\alpha} \ddot{\alpha}+D_{\alpha} \dot{\alpha}+M(\alpha)=M_{e \alpha}
\end{array}\right.
$$

where $L=\rho V^{2} b c_{l}$ and $M_{e \alpha}=2 \rho V^{2} b^{2} c_{m}$ are the aerodynamic lift and moment about the elastic axis, respectively, $c_{l}$ is the lift coefficient, $c_{m}$ is the aerodynamic moment coefficient, and $\rho$ is the air density.

Introducing non-dimensional time $\tau=\omega_{\alpha} t$ and mass ratio $\mu=m / \pi \rho b^{2}$, Eq. (3) can be written as

$$
\left\{\begin{array}{l}
\frac{h^{\prime \prime}}{b}+x_{\alpha} \alpha^{\prime \prime}+2 \zeta_{h}\left(\frac{\omega_{h}}{\omega_{\alpha}}\right) \frac{h^{\prime}}{b}+\left(\frac{\omega_{h}}{\omega_{\alpha}}\right)^{2} \frac{h}{b}=\frac{U^{2}}{\pi \mu}\left(-c_{l}\right) \\
x_{\alpha} \frac{h^{\prime \prime}}{b}+r_{\alpha}^{2} \alpha^{\prime \prime}+2 \zeta_{\alpha} r_{\alpha}^{2} \alpha^{\prime}+r_{\alpha}^{2} \alpha=\frac{U^{2}}{\pi \mu}\left(2 c_{m}\right)-r_{\alpha}^{2} f_{n o n}
\end{array}\right.
$$

where $(\cdot)^{\prime}=d(\cdot) / d \tau,(\cdot)^{\prime \prime}=d^{2}(\cdot) / d \tau^{2}$, and $U=V / b \omega_{\alpha}$ is the non-dimensional airspeed. Subsequently, the governing equation can be written in matrix form,

$$
\boldsymbol{M} \boldsymbol{\xi}^{\prime \prime}+\boldsymbol{D} \boldsymbol{\xi}^{\prime}+\boldsymbol{K} \boldsymbol{\xi}=\frac{U^{2}}{\pi \mu} \boldsymbol{f}_{a}+\boldsymbol{F}_{n o n}
$$

where $\boldsymbol{M}=\left[\begin{array}{cc}1 & x_{\alpha} \\ x_{\alpha} & r_{\alpha}^{2}\end{array}\right]$ is the mass matrix, $\boldsymbol{D}=\left[\begin{array}{cc}2 \zeta_{h}\left(\frac{\omega_{h}}{\omega_{\alpha}}\right) & 0 \\ 0 & 2 \zeta_{\alpha} r_{\alpha}^{2}\end{array}\right]$ is the damping matrix, $\boldsymbol{K}=\left[\begin{array}{cc}\left(\frac{\omega_{h}}{\omega_{\alpha}}\right)^{2} & 0 \\ 0 & r_{\alpha}^{2}\end{array}\right]$ is the stiffness matrix, and $\boldsymbol{F}_{\text {non }}=\left\{\begin{array}{ll}0 & -r_{\alpha}^{2} f_{\text {non }}\end{array}\right\}^{T}$ is the nonlinear term induced by the nonlinear spring in pitching DOF. For this aeroelastic system, $\boldsymbol{\xi}=\left\{\begin{array}{lll}h / b & \alpha\end{array}\right\}^{T}$ and 
$\boldsymbol{f}_{a}=\left\{\begin{array}{ll}-c_{l} & 2 c_{m}\end{array}\right\}^{T}$ serve as the generalized displacements and the generalized aerodynamic forces, respectively. The generalized aerodynamic forces corresponding to the generalized displacements in transonic air flow can be obtained from full-scale CFD simulation or the aerodynamic ROM, which are described in the following sections.

By defining the structural state vector $\boldsymbol{x}_{s}=\left\{\begin{array}{ll}\xi & \xi^{\prime}\end{array}\right\}^{T}$, the governing equations of the aeroelastic system can be written as

$$
\boldsymbol{x}_{s}^{\prime}=\boldsymbol{A}_{s} \boldsymbol{x}_{s}+\frac{U^{2}}{\pi \mu} \boldsymbol{B}_{s} \boldsymbol{f}_{a}+\boldsymbol{B}_{s} \boldsymbol{F}_{\text {non }}
$$

where $\boldsymbol{A}_{s}=\left[\begin{array}{cc}\mathbf{0} & \boldsymbol{I} \\ -\boldsymbol{M}^{-1} \boldsymbol{K} & -\boldsymbol{M}^{-1} \boldsymbol{D}\end{array}\right]$, and $\boldsymbol{B}_{s}=\left[\begin{array}{c}\mathbf{0} \\ \boldsymbol{M}^{-1}\end{array}\right]$.

\subsection{Time marching approach}

\subsubsection{Full-scale CFD simulation}

As mentioned in section 1, the time marching approach based on CFD technique is a high fidelity tool to calculate the aeroelastic response in transonic air flow. Nowadays many commercial software packages are capable of conducting fluid-structure interaction simulations directly or via user-defined functions. In the current investigation, Fluent is used to carry out the aeroelastic response due to its high flexibility of using User-Defined Function (UDF) to incorporate with the structural model in CFD simulation.

Fluent is a general purpose CFD program, which can be used to model a wide range of incompressible and compressible air flow. In the present study, the pressure-based coupled algorithm is applied to solve the fluid governing equations. In Fluent, a control-volume-based technique is employed to convert the general scalar transport equation to an algebraic equation, which is solved 
by using a point implicit (Gauss-Seidel) linear equation solver in conjunction with an algebraic multigrid (AMG) method. For dealing with viscous flow problems, a classical one-equation turbulence model, the S-A model, is used here. For spatial discretization, the second-order upwind scheme is utilized to interpolate the convection terms. In terms of temporal discretization, a technique called bounded second order implicit time integration is employed in Fluent for real-time advancement.

The CFD simulation for viscous flow usually requires a high mesh resolution, which may limit the movement of the airfoil. A Radial Basis Functions $(\mathrm{RBF})$ interpolation for large mesh deformation [21] is implemented to enhance the capability of mesh deformation in ANSYS Fluent via user-defined function (UDF). The RBF interpolation $s(\boldsymbol{x})$, representing the displacement of the CFD mesh [21, 22], can be expressed by a sum of basis functions

$$
s(\boldsymbol{x})=\sum_{i=1}^{i=n_{b}} \alpha_{i} \phi\left(\left\|\boldsymbol{x}-\boldsymbol{x}_{b i}\right\|\right)
$$

where $\phi$ is the basis function, $\boldsymbol{x}_{b i}$ is the center of the RBF describing the displacement of the boundary nodes, $n_{b}$ is the number of boundary nodes, $\|\cdot\|$ is the norm biasing,

$$
\left\|\boldsymbol{x}-\boldsymbol{x}_{\boldsymbol{i}}\right\|=\sqrt{\left(x-x_{i}\right)^{2}+\left(y-y_{i}\right)^{2}} / R
$$

where $R$ is the support radius, and $R=10 c$ is applied herein. Wendland's C2 function is implemented for the basis function

$$
\phi(\|\boldsymbol{x}\|)=\left\{\begin{array}{cc}
(1-\|\boldsymbol{x}\|)^{4}(4\|\boldsymbol{x}\|+1) & \|\boldsymbol{x}\|<1 \\
0 & \|\boldsymbol{x}\| \geq 1
\end{array}\right.
$$


When the motion of nodes on boundary, i.e. $\boldsymbol{d}_{b}$, is specified, coefficients $\boldsymbol{\alpha}=\left\{\alpha_{1}, \ldots, \alpha_{n b}\right\}^{T}$ can be obtained by the inverse of Eq. (7),

$$
\boldsymbol{\alpha}=\boldsymbol{d}_{b} M_{b, b}^{-1}
$$

where $\boldsymbol{M}_{b, b}$ is an $n_{b} \times n_{b}$ matrix containing the evaluations of the basis function $\phi_{b_{i} b_{j}}=\phi\left(\left\|\boldsymbol{x}_{b_{i}}-\boldsymbol{x}_{b_{j}}\right\|\right)$. Then the displacement of all remaining nodes can be determined by Eq. (7).

\subsubsection{Aerodynamic ROM in transonic flow}

Among the numerous available methods, system identification is an effective and efficient technique to establish an aerodynamic ROM. Following the suggestion from Refs. [23, 24], the autoregressive moving average (ARX) model is used to establish the ROM of transonic aerodynamics in both inviscid and viscous air flow. The time domain equation for multi-input and multi-output (MIMO) ARX model can be described as

$$
\boldsymbol{f}_{\boldsymbol{a}}(k)=\sum_{i=1}^{n a} \boldsymbol{A}_{\boldsymbol{i}} \boldsymbol{f}_{\boldsymbol{a}}(k-i)+\sum_{i=0}^{n b-1} \boldsymbol{B}_{\boldsymbol{i}} \boldsymbol{\xi}(k-i)
$$

The ARX model is easy to establish the ROM mathematically, because the system response at any time step $\boldsymbol{f}_{\boldsymbol{a}}(k)$ is just a linear combination of past inputs $\boldsymbol{\xi}(k-i)$ and outputs $\boldsymbol{f}_{\boldsymbol{a}}(k-i)$ of the model. With an assumed model order consisting of $n a$ past outputs and $n b$ inputs, the only task is to identify the constant coefficient matrices $\boldsymbol{A}_{\boldsymbol{i}}$ and $\boldsymbol{B}_{\boldsymbol{i}}$.

In the present study, a so-called "3211" signal used by Cowan et al. [23] is utilized as the input of the CFD solve due to its ease of implementation and broad frequency spectra. The least squares method is applied to fit the time 
history of the output of the CFD solver, i.e., $\boldsymbol{f}_{\boldsymbol{a}}$, to carry out the unknown coefficient matrices in Eq.(11).

One challenging problem of using an ARX model to build aerodynamic ROM is to identify the model order, especially for transonic viscous air flow due to its complicated unsteady characteristics. In theory, the order of aerodynamic ROM could vary at different flow conditions, for instance, Mach number and flow viscosity. In the present study, identifying the model order is treated as a minimization problem of the flow condition, which can be written in the general form of an optimization problem,

$$
\begin{aligned}
& \min _{\substack{n a=1 . .12 \\
n b=1 \ldots 12}} J \\
& \text { where } \quad J=w\left\|c_{l, C F D}-c_{l, R O M}\right\|+(1-w)\left\|c_{m, C F D}-c_{m, R O M}\right\|
\end{aligned}
$$

where $w$ is the weight factor, chosen from 0.2 to 0.4 herein.

This is a mixed variable, non-smooth and nonlinear problem, hence a genetic algorithm (GA) can be implemented to search the most appropriate order of ARX. With well-determined orders and corresponding coefficient matrix, the discrete-time ARX model can be transformed into the continuoustime form through the Tustin approximation [25]. 


\subsubsection{Aerodynamic interpolation technique}

Applying the classical fourth-order Runge-Kutta (RK4) to Eq.(6) yields

$$
\left\{\begin{aligned}
& \boldsymbol{x}_{s, n+1}=\boldsymbol{x}_{s, n}+\left(\boldsymbol{k}_{1}+2 \boldsymbol{k}_{2}+2 \boldsymbol{k}_{3}+\boldsymbol{k}_{4}\right) / 6 \\
& \boldsymbol{k}_{1}=\Delta \tau\left(\boldsymbol{A}_{s} \boldsymbol{x}_{s, n}+\frac{U^{2}}{\pi \mu} \boldsymbol{B}_{s} \boldsymbol{f}_{a}\left(\boldsymbol{x}_{s, n}, \tau\right)+\boldsymbol{B}_{s} \boldsymbol{F}_{n o n}\left(\boldsymbol{x}_{s, n}\right)\right) \\
& \boldsymbol{k}_{2}=\Delta \tau\left(\boldsymbol{A}_{s}\left(\boldsymbol{x}_{s, n}+\boldsymbol{k}_{1} / 2\right)+\frac{U^{2}}{\pi \mu} \boldsymbol{B}_{s} \boldsymbol{f}_{a}\left(\boldsymbol{x}_{s, n}+\boldsymbol{k}_{1} / 2, \tau+\Delta \tau / 2\right)\right. \\
&\left.\quad+\boldsymbol{B}_{s} \boldsymbol{F}_{n o n}\left(\boldsymbol{x}_{s, n}+\boldsymbol{k}_{1} / 2\right)\right) \\
& \boldsymbol{k}_{3}=\Delta \tau\left(\boldsymbol{A}_{s}\left(\boldsymbol{x}_{s, n}+\boldsymbol{k}_{2} / 2\right)+\frac{U^{2}}{\pi \mu} \boldsymbol{B}_{s} \boldsymbol{f}_{a}\left(\boldsymbol{x}_{s, n}+\boldsymbol{k}_{2} / 2, \tau+\Delta \tau / 2\right)\right. \\
&\left.\quad+\boldsymbol{B}_{s} \boldsymbol{F}_{n o n}\left(\boldsymbol{x}_{s, n}+\boldsymbol{k}_{2} / 2\right)\right) \\
& \boldsymbol{k}_{4}= \Delta \tau\left(\boldsymbol{A}_{s}\left(x_{s, n}+\boldsymbol{k}_{3}\right)+\frac{U^{2}}{\pi \mu} \boldsymbol{B}_{s} \boldsymbol{f}_{a}\left(\boldsymbol{x}_{s, n}+\boldsymbol{k}_{3}, \tau+\Delta \tau\right)\right. \\
&+\boldsymbol{B}_{s} \boldsymbol{F}_{n o n}\left(\boldsymbol{x}_{s, n}+\boldsymbol{k}_{3}\right)
\end{aligned}\right.
$$

As we know, the aerodynamic forces are determined by time and airfoil motion in time marching CFD simulations. Due to lack of the information of displacement at time $\tau+\Delta \tau / 2$, it is impossible to carry out evaluations of the generalized aerodynamic forces like $\boldsymbol{f}_{a}\left(\boldsymbol{x}_{s, n}+\boldsymbol{k}_{1} / 2, \tau+\Delta \tau / 2\right)$ in Eq. (12). However, it is reasonable to assume that the aerodynamic forces are continuously changing over time. Therefore, theoretically speaking, it is feasible to predict the aerodynamic forces by implementing an interpolation method of $\boldsymbol{f}_{a}$ but neglecting the influence of $\boldsymbol{x}$ and $\boldsymbol{k}_{i}$. An RK4 scheme with aerodynamic interpolation technique can be derived from Eq. (12), which reduces 
to

$$
\left\{\begin{aligned}
\boldsymbol{x}_{s, n+1}=\boldsymbol{x}_{s, n}+\left(\boldsymbol{k}_{1}+2 \boldsymbol{k}_{2}+2 \boldsymbol{k}_{3}+\boldsymbol{k}_{4}\right) / 6 \\
\boldsymbol{k}_{1}=\Delta \tau\left(\boldsymbol{A}_{s} \boldsymbol{x}_{s, n}+\frac{U^{2}}{\pi \mu} \boldsymbol{B}_{s} \boldsymbol{f}_{a}\left(\boldsymbol{x}_{s, n}, \tau\right)+\boldsymbol{B}_{s} \boldsymbol{F}_{n o n}\left(\boldsymbol{x}_{s, n}\right)\right) \\
\boldsymbol{k}_{2}=\Delta \tau\left(\boldsymbol{A}_{s}\left(\boldsymbol{x}_{s, n}+\boldsymbol{k}_{1} / 2\right)+\frac{U^{2}}{\pi \mu} \boldsymbol{B}_{s} \boldsymbol{f}_{a}(\tau+\Delta \tau / 2)\right. \\
\left.\quad+\boldsymbol{B}_{s} \boldsymbol{F}_{n o n}\left(\boldsymbol{x}_{s, n}+\boldsymbol{k}_{1} / 2\right)\right) \\
\boldsymbol{k}_{3}=\Delta \tau\left(\boldsymbol{A}_{s}\left(\boldsymbol{x}_{s, n}+\boldsymbol{k}_{2} / 2\right)+\frac{U^{2}}{\pi \mu} \boldsymbol{B}_{s} \boldsymbol{f}_{a}(\tau+\Delta \tau / 2)\right. \\
\left.\quad+\boldsymbol{B}_{s} \boldsymbol{F}_{n o n}\left(\boldsymbol{x}_{s, n}+\boldsymbol{k}_{2} / 2\right)\right) \\
\boldsymbol{k}_{4}=\Delta \tau\left(\boldsymbol{A}_{s}\left(x_{s, n}+\boldsymbol{k}_{3}\right)+\frac{U^{2}}{\pi \mu} \boldsymbol{B}_{s} \boldsymbol{f}_{a}(\tau+\Delta \tau)\right. \\
\quad+\boldsymbol{B}_{s} \boldsymbol{F}_{n o n}\left(\boldsymbol{x}_{s, n}+\boldsymbol{k}_{3}\right)
\end{aligned}\right.
$$

where $\boldsymbol{f}_{a}(\tau+\Delta \tau / 2)$ and $\boldsymbol{f}_{a}(\tau+\Delta \tau)$ can be obtained by using a second order interpolation on the aerodynamic forces at previous time steps $\boldsymbol{f}_{a}(\tau)$, $\boldsymbol{f}_{a}(\tau-\Delta \tau)$ and $\left.\boldsymbol{f}_{a}(\tau-2 \Delta \tau)\right)$.

\subsubsection{Henon method}

The structural restoring moment for free-play Eq.(1) is a piecewise linear function with three linear sub-domains divided by the discontinuity crossover $( \pm \delta)$ of the integration step according to the location $\alpha\left(t_{n}\right)$, as shown in Fig. 2. In order to switch the integration in different linear sub-domains precisely, the prediction for the crossover of the integration should be accurate. The application of the classical fourth-order Runge-Kutta (RK4) method is limited due to the numerical inaccuracy induced by the crossover of the integration step according to Ref. [26]. Dai et al. [26] also pointed out that one effective method for accurately detecting the crossover is proposed by Henon [27], herein called the Henon method, originally applied to construct Poincaré maps.

The main idea of Henon method is described as follows [26, 27]. The 


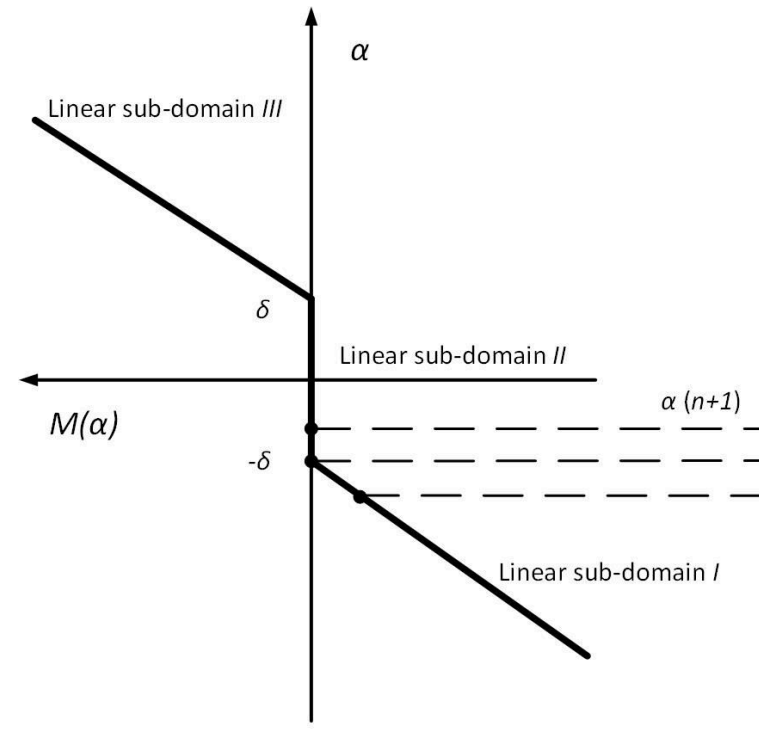

Structural restoring moment for free-play

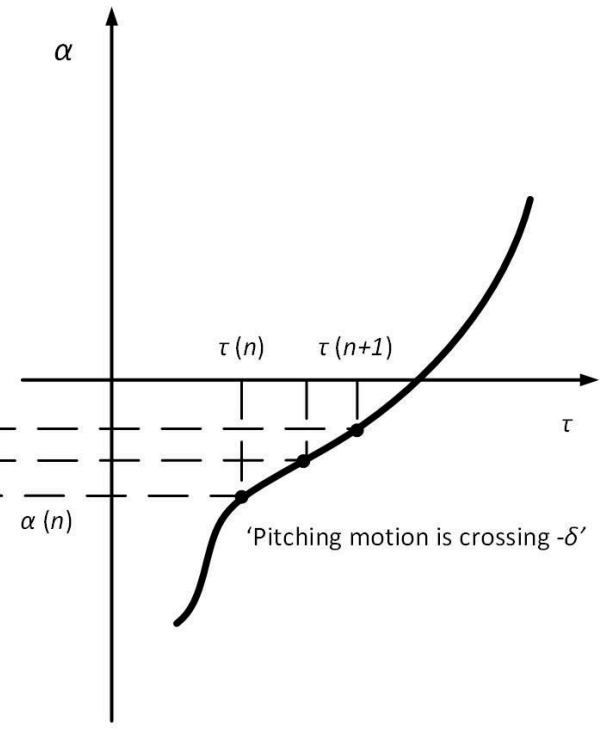

Time history of pitching motion

Figure 2: Sketch of switch from different linear sub-domain in integration procedure

governing equation of the aeroelastic system, Eq.(6), can be rewritten as

$$
\frac{d}{d \tau}\left\{\begin{array}{c}
x_{1} \\
x_{2} \\
\vdots \\
x_{n}
\end{array}\right\}=\left\{\begin{array}{c}
f_{1}(\boldsymbol{x}) \\
f_{2}(\boldsymbol{x}) \\
\vdots \\
f_{n}(\boldsymbol{x})
\end{array}\right\}
$$

where $x_{2}$ stands for the pitching motion $\alpha$. As shown in Fig. 2, when the integration is switching from one sub-domain to another one, i.e., pitching motion crosses $\delta$ or $-\delta$, exchange the dependent variable $\alpha$ with independent variable $\tau$, which will be shown below in detail. Since the distance between previous location $\alpha\left(\tau_{n-1}\right)$ and discontinuity crossover $(\delta$ or $-\delta)$ is known, the response of the aeroelastic system can be integrated from its previous location forward to the exact point of discontinuity within one step. Then 
time is reverted to $\tau$, and the classical RK4 is then applied to integrate with the new linear sub-domain until a next discontinuity is detected.

Detail of the steps of exchanging $\alpha$ with $\tau$ are described as follows. When switching of the linear sub-domain takes place, exchange the dependent variable $\alpha$ and independent variable $\tau$, which is realized via first dividing each of the equation of Eq. (14) by $d x_{2} / d \tau=f_{2}(\boldsymbol{x})$. And then replace the first equation by $d \tau / d x_{2}=1 / f_{2}(\boldsymbol{x})$. Consequently, a new system with $x_{2}$ as the independent variable is obtained.

$$
\frac{d}{d x_{2}}\left\{\begin{array}{c}
x_{1} \\
\tau \\
\vdots \\
x_{n}
\end{array}\right\}=\left\{\begin{array}{c}
f_{1}(\boldsymbol{x}) / f_{2}(\boldsymbol{x}) \\
1 / f_{2}(\boldsymbol{x}) \\
\vdots \\
f_{n}(\boldsymbol{x}) / f_{2}(\boldsymbol{x})
\end{array}\right\}
$$

Note that the new system is used only for one integration step immediately before $\alpha$ crosses $\delta$ or $-\delta$.

In the following sections, RK4 with Henon method is applied to obtain the nonlinear aeroelastic response as long as the free-play nonlinearity is considered in the structural model.

\subsection{Eigenvalue analysis for linear flutter solution}

With well-determined orders of aerodynamic ROM by GA and corresponding coefficient matrix, i.e. $\boldsymbol{A}_{\boldsymbol{i}}$ and $\boldsymbol{B}_{\boldsymbol{i}}$, the discrete-time ARX model can be transformed into the continuous-time form through Tustin approximation [25], which can be described in state-space form as

$$
\left\{\begin{array}{l}
x_{a}^{\prime}=A_{a} x_{a}+B_{a} \xi \\
f_{a}=C_{a} x_{a}+D_{a} \xi
\end{array}\right.
$$


where the aerodynamic state vector is

$$
\begin{aligned}
& \boldsymbol{x}_{\boldsymbol{a}}(k)=\left[\begin{array}{lllll}
\boldsymbol{f}_{\boldsymbol{a}}(\tau-\Delta \tau) & \cdots & \boldsymbol{f}_{\boldsymbol{a}}\left(\tau-n_{a} \Delta \tau\right) & \boldsymbol{\xi}(\tau-\Delta \tau) & \cdots
\end{array}\right. \\
& \left.\boldsymbol{\xi}\left(\tau-\left(n_{b}-1\right) \Delta \tau\right)\right]^{T}
\end{aligned}
$$

Regardless the nonlinear structural term of $\boldsymbol{F}_{\text {non }}$ in Eq. (6), introducing $\boldsymbol{x}=\left\{\begin{array}{ll}\boldsymbol{x}_{\boldsymbol{s}}^{T} & \boldsymbol{x}_{\boldsymbol{a}}^{T}\end{array}\right\}^{T}$ and coupling the structural motion with the aerodynamic ROM of Eq.(16), the governing equation for the aeroelastic system in statespace form is obtained,

$$
\boldsymbol{x}^{\prime}=\boldsymbol{A} \boldsymbol{x}=\left[\begin{array}{cc}
\boldsymbol{A}_{s}+\frac{U^{2}}{\pi \mu} \boldsymbol{B}_{s} D_{a} C_{s} & \frac{U^{2}}{\pi \mu} B_{s} C_{a} \\
B_{a} C_{s} & A_{a}
\end{array}\right] \boldsymbol{x}
$$

Then, the linear non-dimensional flutter speed $U$ and flutter frequency ratio $\omega / \omega_{\alpha}$ can be obtained by conventional stability analysis, i.e., solving the eigenvalue of $\boldsymbol{A}$ in Eq. (18) at different air speeds.

\subsection{Aerodynamic influence coefficient}

To seek a better understanding of the unsteady characteristic of the aerodynamic model, the aerodynamic influence coefficient is employed in the present study. Based on the authors' previous work [6] [28], CFD or ROM can be regarded as an implicit system with the generalized displacements as inputs and the generalized aerodynamic forces as output. The aerodynamic influence coefficient is the ratio of the first order harmonic component of the complex output and the harmonic input.

According to the definition of aerodynamic influence coefficient, the input of CFD or ROM, i.e. pitching or (plunging) motion of the airfoil, is taken as a sinusoid function,

$$
\alpha(t)=\alpha_{0} \sin (\omega t)
$$


where $\alpha_{0}$ denotes the amplitude and $\omega$ is the frequency. The corresponding output of the CFD solver or aerodynamic ROM, taking aerodynamic moment coefficient as example, is periodic which can be expanded by Fourier series. Only the first order harmonic component of the aerodynamic moment coefficient is retained, and it can be written as

$$
c_{m}(t)=\left(c_{m}\right)_{c} \cos (\omega t)+\left(c_{m}\right)_{s} \sin (\omega t)=\left(c_{m}\right)_{c s} \sin (\omega t+\varphi)
$$

The coefficients $\left(c_{m}\right)_{c}$ and $\left(c_{m}\right)_{s}$ can be obtained by fitting the time history of aerodynamic moment coefficient to the above equation. The aerodynamic influence coefficient can be expressed as

$$
Q_{m \alpha}=\frac{2\left(c_{m}\right)_{c s}}{\alpha_{0}} e^{i \varphi}=\frac{2\left(c_{m}\right)_{c s}}{\alpha_{0}}[\cos (\varphi)+i \sin (\varphi)]=\Re\left(Q_{m \alpha}\right)+i \Im\left(Q_{m \alpha}\right)
$$

The detailed process to build the aerodynamic influence coefficient can be found in Ref. [28], and the generalized aerodynamic forces corresponding to the airfoil motion can be written as

$$
\left\{\begin{array}{c}
-c_{l} \\
2 c_{m}
\end{array}\right\}=\left[\begin{array}{ll}
Q_{l h} & Q_{l \alpha} \\
Q_{m h} & Q_{m \alpha}
\end{array}\right]\left\{\begin{array}{c}
\frac{h}{b} \\
\alpha
\end{array}\right\}=\boldsymbol{Q}\left\{\begin{array}{c}
\frac{h}{b} \\
\alpha
\end{array}\right\}
$$

where $\boldsymbol{Q}$ is the aerodynamic influence coefficient matrix, which is related to Mach number and reduced frequency $k=\omega b / V$. It should be noted that the amplitudes of motion, namely $\alpha_{0}$ and $h_{0} / b$, should be set to be sufficiently small in CFD calculations. In the aerodynamic ROM method, the steady lift slope $c_{l \alpha}$ and aerodynamic moment coefficient slope $c_{m \alpha}$ can be derived from the real part of the aerodynamic influence coefficient of $Q_{l \alpha}$ and $Q_{m \alpha}$ when the reduced frequency $k$ of pitching motion is sufficiently small. 
Table 1: Grid parameters for the C-type structured mesh for inviscid flow simulation

\begin{tabular}{cccc}
\hline \hline Mesh no & airfoil surface & Radial points & Wake \\
\hline Mesh1 & 101 & 23 & 28 \\
Mesh2 & 149 & 34 & 41 \\
Mesh3 & 221 & 51 & 51 \\
\hline \hline
\end{tabular}

\section{Computational validations}

The aeroelastic airfoil studied here is taken from the work of Kousen and Bendiksen [4], in which a free-play nonlinearity is assumed in the pitching DOF. The airfoil of this model is NACA 64A010, and the relevant parameters are $x_{\alpha}=0.2, r_{\alpha}^{2}=0.29, \mu=60, \omega_{h} / \omega_{\alpha}=0.34335, a=-0.2, \delta=1^{\circ}$. The computed Mach number is 0.87 . Note that the viscous damping from the structure is ignored in the original study.

\subsection{Mesh independence study}

Three computational grids for inviscid air flow simulation with different mesh resolutions are generated to perform the mesh convergence analysis. A C-type mesh is applied as shown in Fig. 3, and the outer boundary of the computational domain extends to a distance of $50 \mathrm{c}$ from the airfoil as shown in Fig. 4. Detailed grid parameters for these three computational meshes are presented in Table 1.

CFD simulations of a NACA 64A010 airfoil with a sinusoid oscillating motion for $\alpha_{0}=1^{\circ}$ and $k=0.1$ at Mach 0.87 are carried out. The time history of the unsteady aerodynamic coefficient for different meshes is shown in Fig. 5. It can be seen that the amplitudes of $c_{l}$ and $c_{m}$ obtained by using 


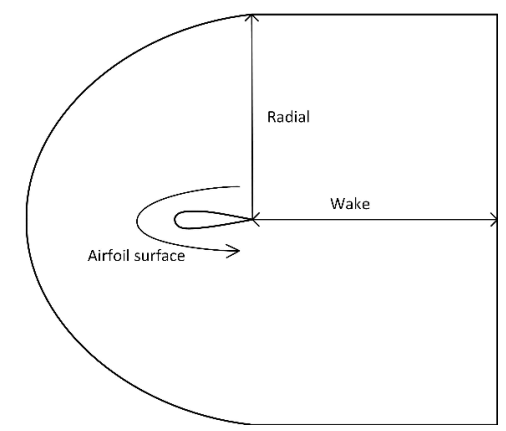

Figure 3: Sketch of C-type CFD mesh

Table 2: Grid parameters for the C-type structured mesh for viscous flow simulation

\begin{tabular}{cccc}
\hline \hline Mesh no & airfoil surface & Radial points & Wake \\
\hline Mesh1 & 293 & 61 & 59 \\
Mesh2 & 441 & 91 & 89 \\
Mesh3 & 663 & 137 & 163 \\
\hline \hline
\end{tabular}

Mesh 1 are a little smaller than those obtained by using Mesh 2 and Mesh 3, but results obtained by using Mesh 2 and Mesh 3 agree well. Thus both Mesh 2 and Mesh 3 are appropriate to calculate the unsteady aerodynamic forces. By considering the computational costs, Mesh 2 with 7524 points is applied in the following sections.

Other C-type meshes with a fine grid resolution are generated for CFD simulation of viscous air flow. Similar to the mesh sensitivity assessment of inviscid flow simulation, three meshes, whose parameters are listed in Table 2, are generated. The outer boundary for the computational domain extends to $50 c$ from the airfoil, and the first layer thickness of all these grids is $1 \times 10^{-5} c$, with $0<y^{+}<1$ on the airfoil surface.

Figure 6 shows the time history of the aerodynamic coefficients for a sinu- 


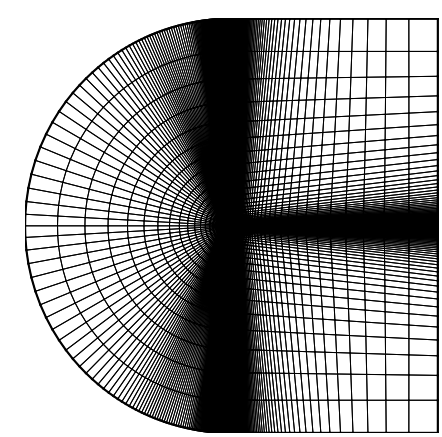

(a)

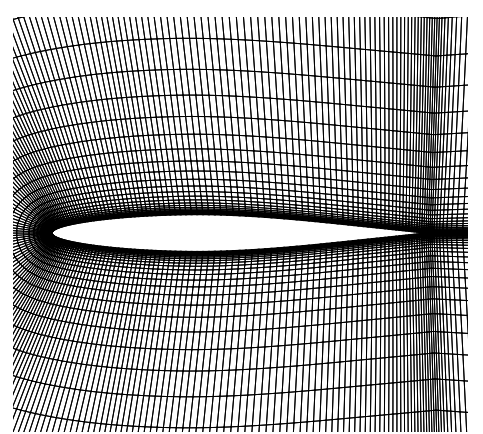

(b)

Figure 4: Computational grids of NACA 64A010 airfoil for inviscid air flow simulation: (a) overall, (b) close-up.

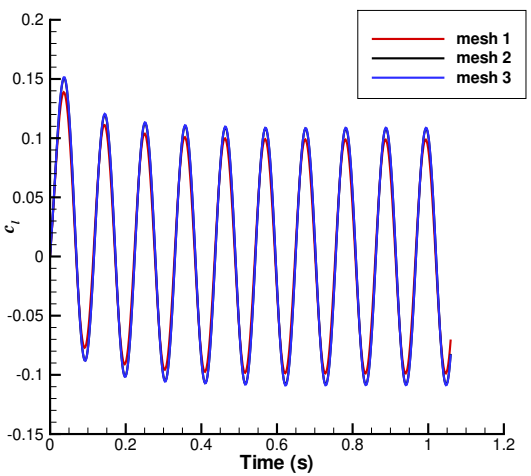

(a)

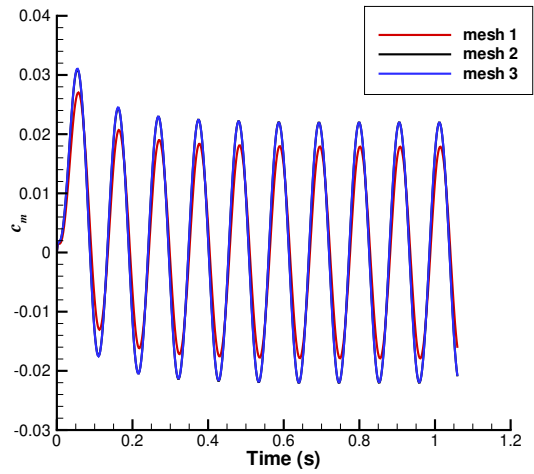

(b)

Figure 5: Comparison of aerodynamic coefficients of NACA 64A010 airfoil from different meshes for inviscid air flow simulation (Mach 0.87, $\alpha_{0}=1^{\circ}, k=0.1$ and $a=-0.6$ ): (a) lift coefficient, (b) aerodynamic moment coefficient. 


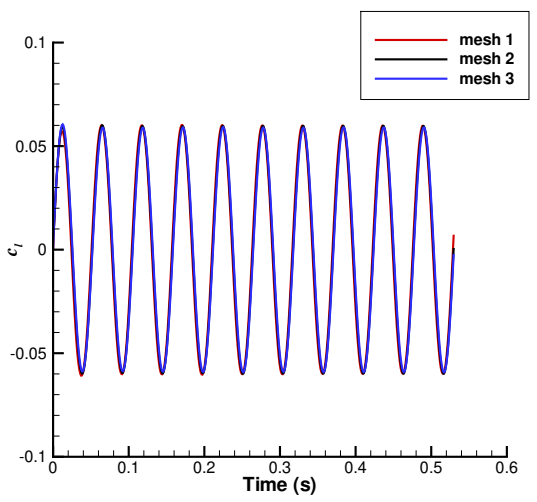

(a)

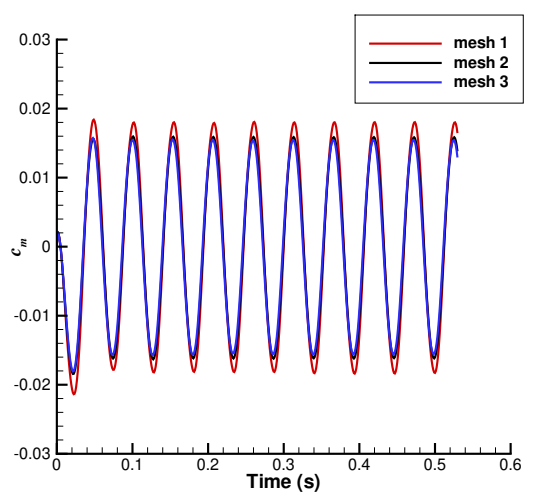

(b)

Figure 6: Comparison of aerodynamic coefficients of NACA 64 A010 airfoil from different meshes for viscous air flow simulation (Mach 0.87, $\alpha_{0}=1^{\circ}, k=0.2$ and $a=-0.6$ ): (a) lift coefficient, (b) aerodynamic moment coefficient.

soid oscillating motion with $\alpha_{0}=1^{\circ}$ and $k=0.1$ at Mach 0.87. Apparently, both Mesh 2 and Mesh 3 meet the requirement for unsteady viscous flow simulations. Considering the computational costs, however, Mesh 2 with 55440 points is applied in the following sections. Note that based on the mesh size, the computational cost for the viscous solution should be over 7 times that of the inviscid solution. Thus, it can be time-consuming to obtain the aeroelastic response by using full CFD simulation for the viscous solution, indicating the necessity of adopting aerodynamic ROM technique in viscous CFD simulation.

\subsection{Time step convergence study}

As noted in our previous study [16], the time-step size in time marching approach with CFD technique has significant influence on the calculated aeroelastic response, especially for the cases considering structural nonlin- 


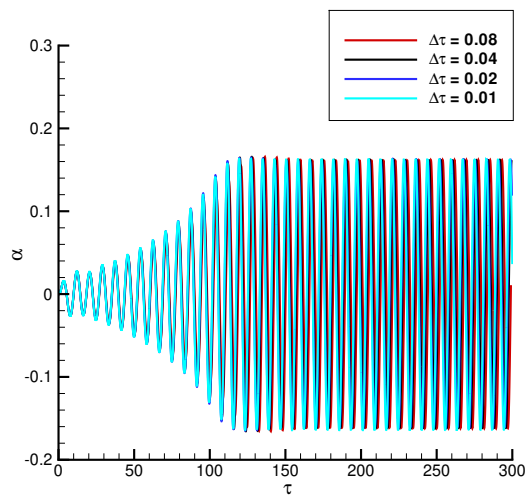

Figure 7: Comparison of calculated time histories of pitching motion for linear structure model in inviscid air flow at Mach 0.87 by using different time step size at $U=3.8$.

earity in transonic air flow. Therefore, time step convergence studies are conducted with a series of time-step sizes for the linear and nonlinear structural model in both inviscid and viscous air flow in this section.

Figure 7 shows the calculated aeroelastic response for linear structure in inviscid air flow obtained by using different time-step sizes. It is found that the aeroelastic system has a simple LCO at $U=3.8$, and the differences between calculated responses from different time steps are small.

Figure 8 shows the computed pitching responses of the aeroelastic model with free-play nonlinearity in inviscid air flow. It is interesting to note that time histories and phase plots of pitching DOF obtained by using different time step size are almost the same. Thus, in terms of time step sensitivity, the good agreements of aeroelastic response from $\Delta \tau=0.2$ to $\Delta \tau=0.01$ reveal the excellent robustness of the present approach. Those phenomena are entirely different from those observed in our previous investigation [16], in which Ansys-CFX solver was applied to calculate the nonlinear response. 


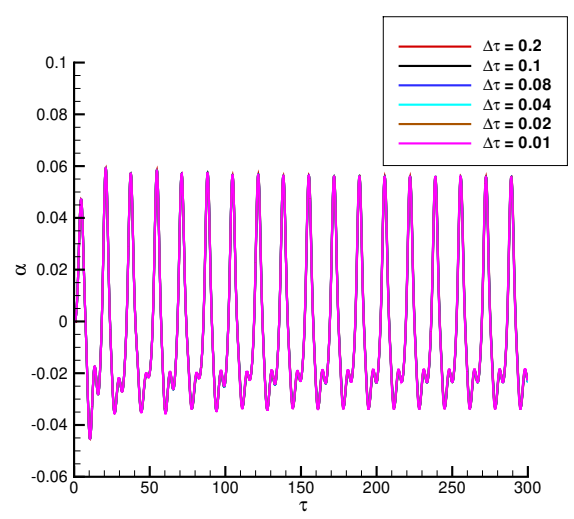

(a)

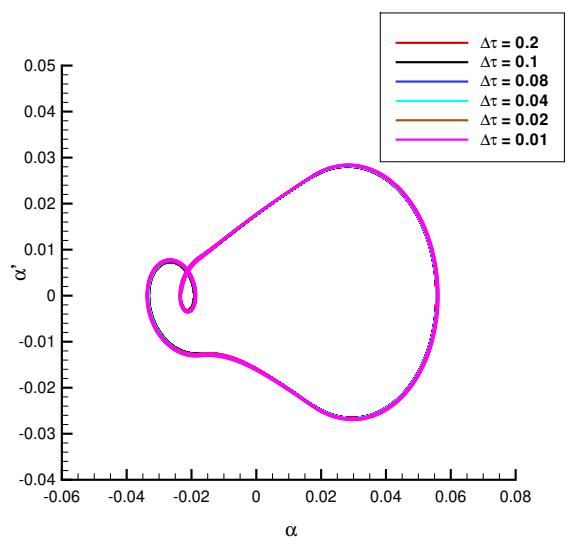

(b)

Figure 8: Comparison of calculated aeroelastic responses for nonlinear structure model in inviscid air flow at Mach 0.87 by using different time step size at $U=1.8$ : (a) time history, (b) phase plot.

Compared to Ansys-CFX solver, the main difference is the implementing of aerodynamic force interpolation technique in the present approach. Our results imply that applying aerodynamic force interpolation technique in the time marching approach based on CFD can reduce the sensitivity to the time step. In summary, the time-step size of $\Delta \tau=0.04$ can be adopted in the following simulations of inviscid flow.

In the same way, the time step independent study is performed for both linear and nonlinear structural aeroelastic airfoil in viscous flow, as shown in Fig. 9. For the case with the linear structure, the calculated aeroelastic response converges to the same LCO with decreasing time-step size. The responses of the nonlinear aeroelastic model are also insensitive to the time step, which is coincident with the case of inviscid air flow. Hence a suitable time step is $\Delta \tau=0.04$ for both linear and nonlinear structural models, which 


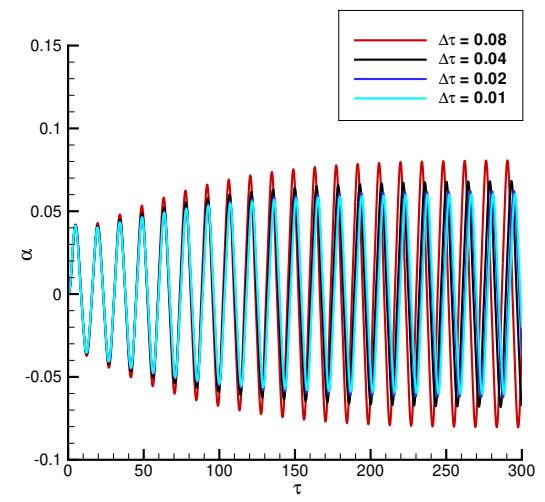

(a)

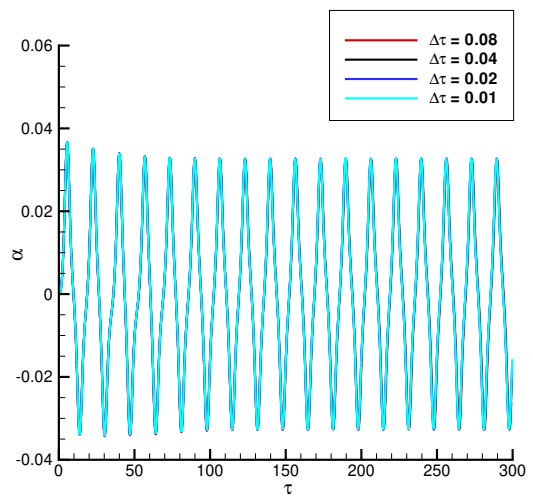

(b)

Figure 9: Comparison of calculated time histories of pitching motion in viscous air flow at Mach 0.87 by using different time step size at: (a) $U=4$ for linear model, (b) $U=1.8$ for nonlinear model.

is applied in the following viscous CFD simulations.

\subsection{Transonic flutter boundary for Isogai model}

To conduct a detailed validation for the ROM method and high-fidelity CFD simulation of the present study, a benchmark for transonic flutter prediction, namely the Isogai wing model [1], is employed in the section. Its parameters are $a=-2.0, x_{\alpha}=1.8, r_{\alpha}^{2}=3.48, \omega_{h} / \omega_{\alpha}=1.0, \mu=60$.

The transonic flutter boundary of Isogai wing model has been widely studied with an inviscid Euler solution. Depicting the flutter speed and frequency versus Mach number, the flutter boundary is illustrated in Fig. 10. The results obtained by both the ROM method and the time marching approach based on CFD are in good agreement with those obtained by using time marching solutions $[29,30,31]$ and the transonic frequency domain method [28]. From Fig. 10(a), it can be seen that flutter speed in the 


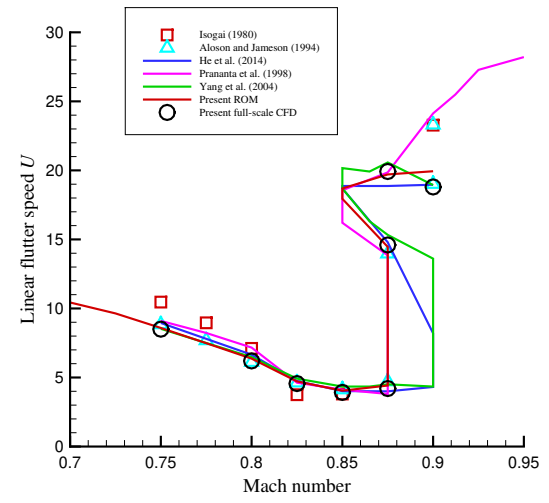

(a)

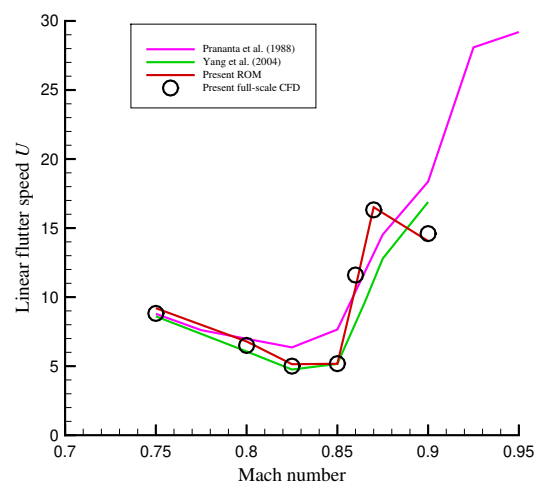

(c)

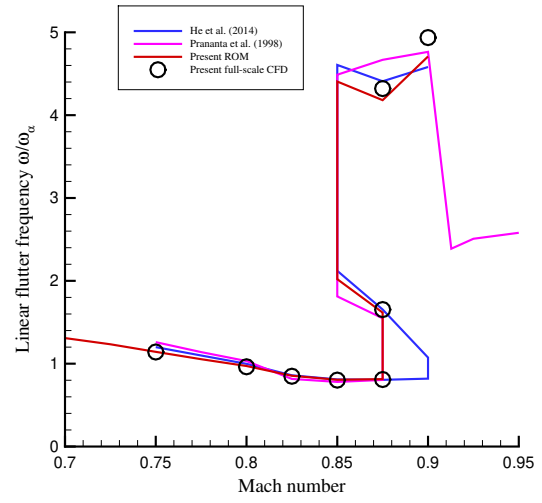

(b)

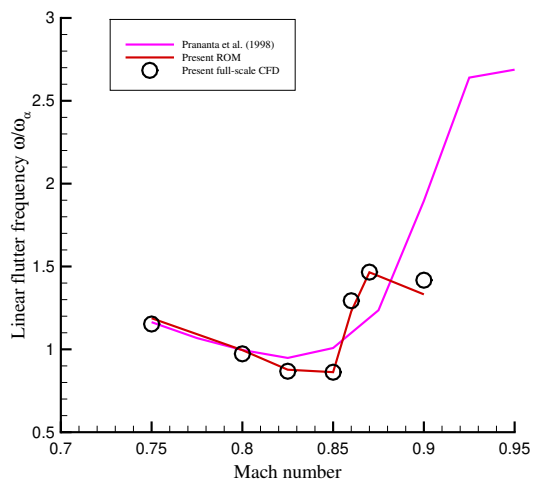

(d)

Figure 10: Transonic flutter boundary of Isogai wing model: (a) flutter speed and (b) flutter frequency for inviscid solution, (c) flutter speed and (d) flutter frequency for viscous solution. 
transonic regime is obviously lower than those in the subsonic regime, which is usually termed as "transonic dip". Moreover, there are multiple values of flutter speed between Mach 0.85 and 0.9 , which forms the so called $S$ shape flutter boundary [32].

Only a few studies of Isogai wing model in viscous flow are available in the existing literature. Aerodynamic ROM method and full-scale CFD simulations are utilized to obtain the flutter solution at different Mach numbers as shown in Fig. 10 (c) and (d). Compared with the existing time marching solutions [30, 31], reasonably good agreements are achieved demonstrating the feasibility of the present methods. It is also found that the so-called $S$ shape flutter boundary observed in the inviscid computational results disappears when aerodynamic viscosity is taken into account. Furthermore, it is found that the significant difference of the flutter solution between Euler and Navier-Stokes calculations occurs at $0.85<M a<0.9$.

\section{Transonic flutter boundary}

In order to obtain the linear flutter characteristic of the aeroelastic airfoil in transonic airflow, a linear structure model is used in this section. In the time marching approach based on CFD, the linear flutter characteristic is obtained by observing the responses at a sequence of successive airspeed. In the ROM method, the linear flutter solution can be obtain by either observing the aeroelastic response or by eigenvalue analysis.

Figure 11 shows the plunging and pitching motion for inviscid solution at Mach 0.87 obtained from time marching approach based on CFD and aerodynamic ROM with a series of airspeeds. As expected, damped, neutrally 


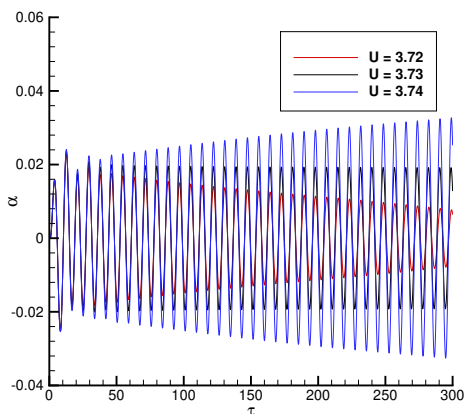

(a)

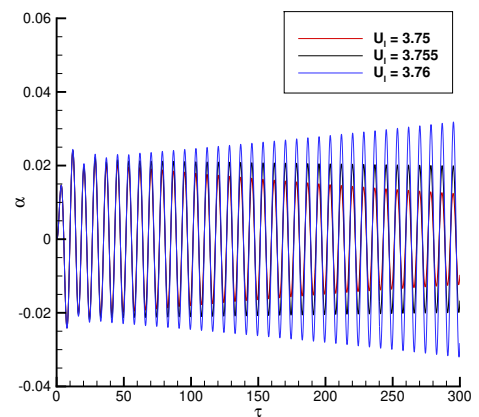

(b)

Figure 11: Aeroelastic response for linear structural model at Mach number 0.87 for inviscid solution from: (a) full-scale CFD simulations and (b) aerodynamic ROM.

stable and divergent motion can be observed as the airspeed successively increases. Hence, the non-dimensional flutter speed and flutter frequency for the inviscid solution is $U=3.73$ and $\omega / \omega_{\alpha}=0.746$ obtained from the full CFD simulation. From the ROM method, the flutter speed and flutter frequency are $U=3.755$ and $\omega / \omega_{\alpha}=0.755$.

Similarly, the aeroelastic response for the viscous solution at different airspeeds is presented in Fig. 12 from both the CFD simulation and the aerodynamic ROM method. The critical flutter condition from the viscous calculation is $U=3.9$ and $\omega / \omega_{\alpha}=0.423$ obtained from CFD simulation, and $U=3.734$ and $\omega / \omega_{\alpha}=0.406$ from the aerodynamic ROM method.

Table 3 summarizes both the non-dimensional flutter speed and flutter frequency ratio obtained by using the different methods for both inviscid and viscous solution at Mach 0.87. Our results from Fluent UDF and ARX model agree well with those obtained by the time marching approach [4] and the Ansys-CFX solver [16]. The difference of the non-dimensional flutter speed 


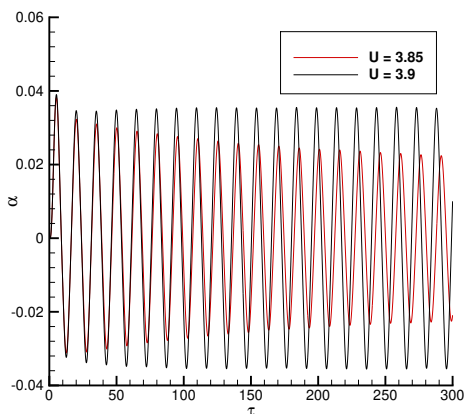

(a)

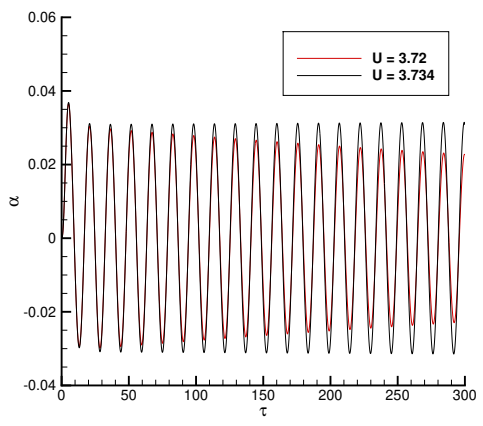

(b)

Figure 12: Aeroelastic response for linear structural model at Mach number 0.87 for viscous solution from: (a) full-scale CFD simulations and (b) aerodynamic ROM.

obtained by using ARX ROM and the time marching method with CFD technique is small, and the flutter frequency ratios are almost the same.

Due to its high efficiency in aerodynamic modeling, the ARX ROM is established over a wide range of Mach number from 0.6 to 0.95. The transonic flutter boundary is achieved by depicting flutter speed and flutter frequency versus Mach number, as shown in Fig. 13. The flutter boundary obtained by full-scale CFD simulation is also presented in Fig. 13 to ensure the accuracy of the flutter solution from the ARX ROM method, in which good agreement is observed. The transonic flutter boundary based on Euler equations obtained by Yang et al. [6] is also plotted for comparison.

From the comparison of transonic flutter boundary of the Isogai wing model in Fig. 10 and the present model in Fig. 13, it is obvious that the effect of flow viscosity on flutter characteristic is different for different Mach number ranges. Regarding the present aeroelastic airfoil, the linear flutter solution considering viscous effects are slightly different from those for the 
Table 3: Comparison of linear flutter speed and flutter frequency at Mach 0.87.

\begin{tabular}{lllllll}
\hline \hline & \multicolumn{2}{l}{ Inviscid air flow } & & \multicolumn{2}{l}{ Viscous air flow } \\
Fluent UDF & $U$ & $\omega / \omega_{\alpha}$ & $U$ & $\omega / \omega_{\alpha}$ \\
ARX ROM & 3.73 & 0.746 & 3.9 & 0.423 \\
Ansys-CFX solver in [16] & 3.95 & 0.861 & 3.938 & - \\
ARMA ROM in [16] & 3.78 & 0.831 & 3.911 & - \\
Kousen and Bendiksen [4] & nearly 4 & - & - & - \\
\hline \hline
\end{tabular}

inviscid results for $M a<0.83$ and $M a>0.91$. However, the flow viscosity has a significant influence on the flutter characteristics, especially for flutter frequency, for $0.84<M a<0.9$.

Similarly to Silva et al. [19], root locus analysis is employed to evaluate aeroelastic behaviors for inviscid and viscous solutions at different Mach number ranges. In the low Mach number range $M a<0.83$, no notable discrepancy of the root locus from the ROM method between Euler and Navier-Stokes based aerodynamics is observed, as shown in Fig. 14.

When the Mach number is increased to $0.84<M a<0.9$, the damping and frequency of the aeroelastic response from the full-scale CFD are also plotted to verify the root locus from the ROM method. A MATLAB-based data post-procedure developed by Bennett and Desmarais [33] is applied to analyze the aeroelastic transients for damping and frequency components. A curve-fitting toolbox in MATLAB is employed to find the best curve fit for the obtained aeroelastic response. From Fig. 15, it is evident that the primary flutter mechanism obtained from the ROM method and the time 


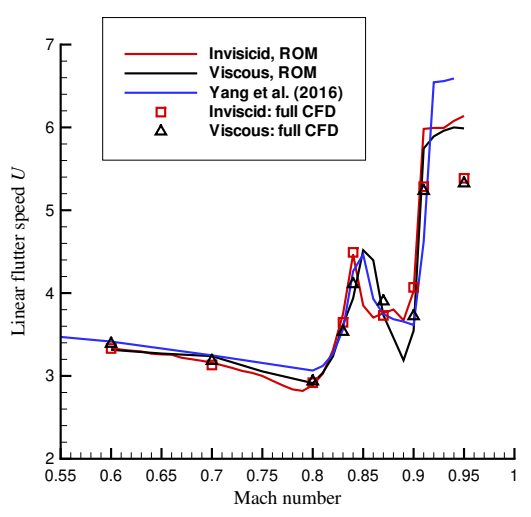

(a)

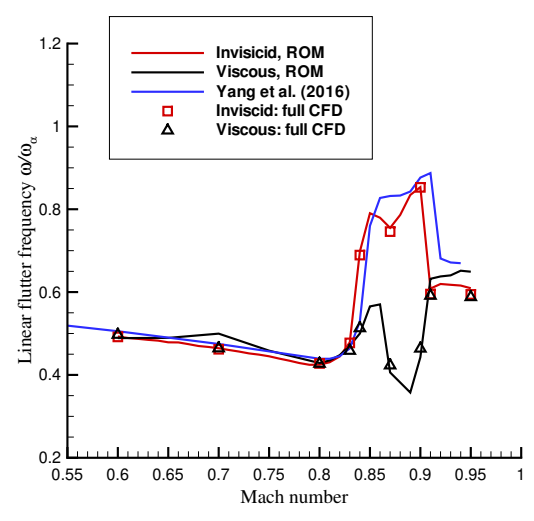

(b)

Figure 13: Comparison of Flutter boundary obtained by inviscid and viscous solutions: (a) flutter speed versus Mach number (b) flutter frequency versus Mach number.

marching approach based on CFD agrees very well. Furthermore, the root locus derived from the Euler equations behaves significantly differently from that for the Navier-Stokes result in this situation. The aeroelastic mode crossing imaginary axis changes from the 2nd mode for the inviscid solution to 1 st mode for the viscous solution. The shift of unstable aeroelastic mode leads to the remarkable flutter frequency difference between inviscid and viscous solutions at this Mach range observed in Fig. 13.

As long as the flow is accelerated further to $0.91<M a<0.95$, the root locus of the aeroelastic system derived from inviscid and viscous aerodynamic models are almost the same, as displayed in Fig. 16. Thus, it is not surprising that the flutter boundary matches well at the high Mach number no matter whether the Euler or Navier-Stokes solver is implemented.

From the above root locus analysis, it can be seen that in the low and high Mach number ranges, aerodynamic viscosity makes little difference to 


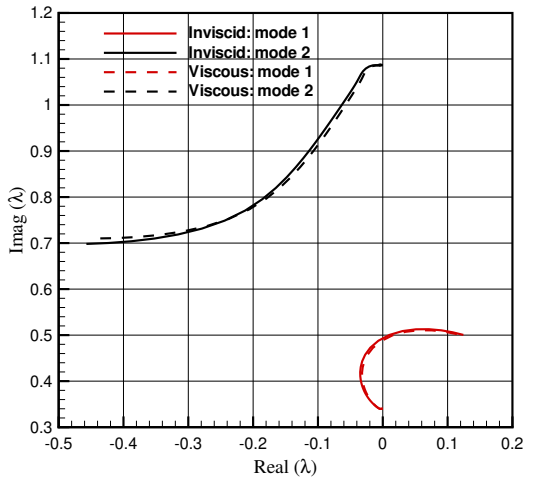

(a)

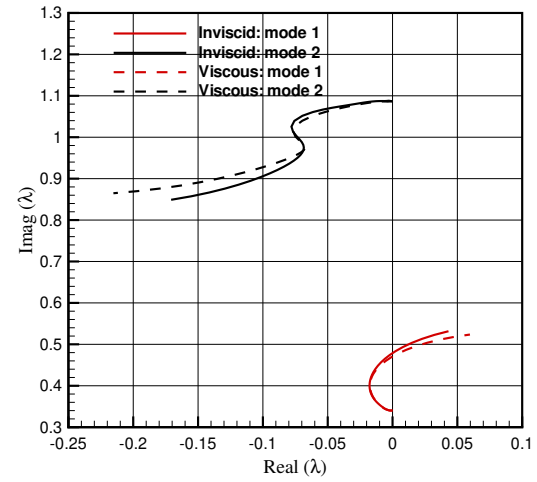

(b)

Figure 14: Aeroelastic root locus from ROM aerodynamics at: (a) Mach 0.6 and (b) Mach 0.83 .

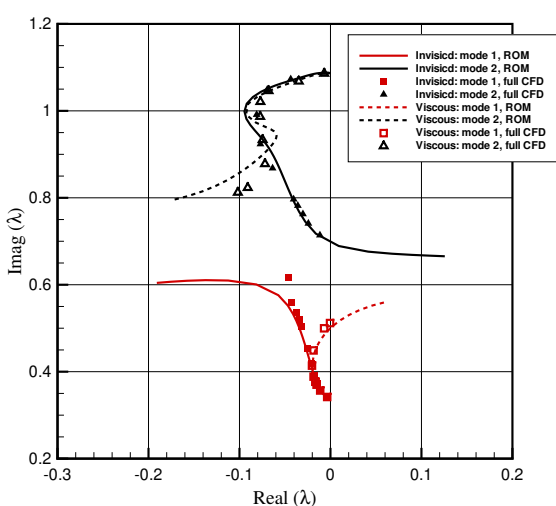

(a)

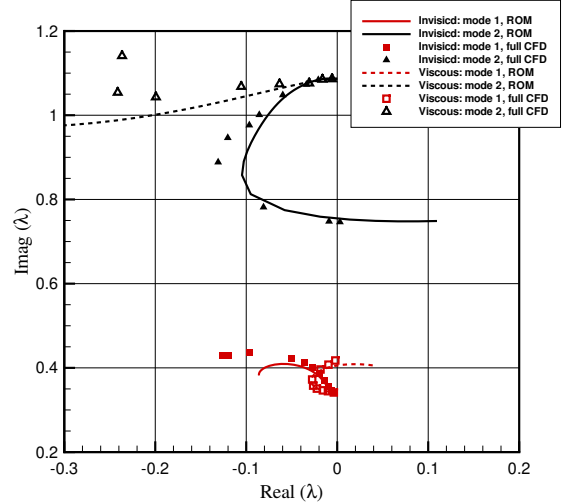

(b)

Figure 15: Aeroelastic root locus from ROM aerodynamics and time marching approach based on CFD at: (a) Mach 0.84 and (b) Mach 0.87. 


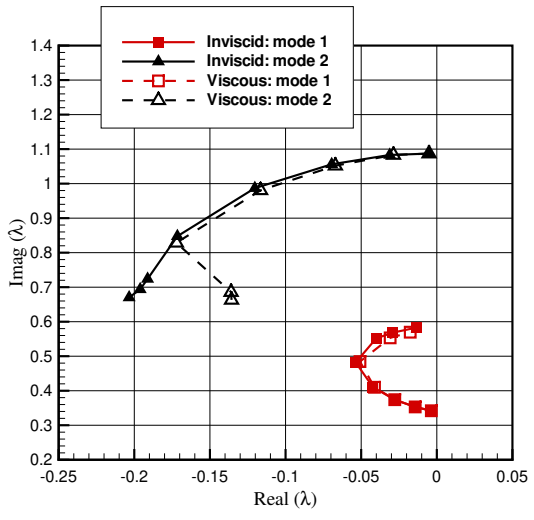

(a)

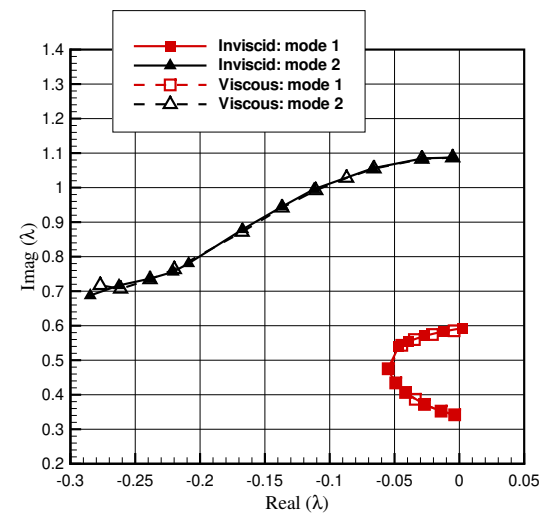

(b)

Figure 16: Aeroelastic root locus from time marching approach based on CFD at: (a) Mach 0.91 and (b) Mach 0.95 .

the flutter solution. But in the special transonic Mach number range, the effect of viscosity can change the unstable aeroelastic mode compared with the inviscid solution. So these results show that the aerodynamic viscosity can affect the unstable mechanism of flutter in transonic regime.

\section{Nonlinear dynamic behaviors in transonic flow}

\subsection{Nonlinear aeroelastic response at Mach 0.87}

To demonstrate the capability to obtain a nonlinear response from Fluent UDF and the ARX model, the typical nonlinear responses are first presented. These responses also show the fundamental nonlinear behavior of the present model in inviscid air flow. Subsequently, the nonlinear behavior in viscous air flow is shown to demonstrate the effects of aerodynamic viscosity.

Figure 17 shows several aeroelastic responses in inviscid airflow at different flow speeds obtained by using Fluent UDF and aerodynamic ROM 
method. Some representative and interesting phenomena, for example, a snap-through phenomenon in Fig. 17 (a) (b), LCOs in Fig. 17 (e) (f) and chaotic motion in Fig. 17 (c) (d), can be captured by both of the two approaches applied in the present study.

Representative phase plots in viscous air flow at different airspeeds are obtained from aerodynamic ROM and CFD simulations as shown in Fig. 18. Simple and complex LCOs can be observed, indicating bifurcation should occur in this Mach number. The good agreement between these two methods indicate the suitability of using aerodynamic ROM to obtain the nonlinear response in transonic viscous air flow.

Figure 19 presents the bifurcation diagram from the ARX model based on Euler solution, showing a double-period bifurcation towards chaos, which agrees well with our previous analysis [16]. These agreements also suggest the feasibility of using Fluent UDF and the ARX ROM method to calculate the nonlinear aeroelastic responses in transonic flow.

Amongst the numerous comparisons of the nonlinear dynamic response from the Euler and Navier-Stokes equations at Mach 0.87, two major differences are summarized. The first is that the snap-through phenomenon happening at a low-speed range in inviscid air flow is no longer observed in the case of viscous flow. Taking the case of $U=0.4$ displayed in Fig. 20 as an example, a damped motion is observed when the Navier-Stokes equations are applied. The other is that the flow speed region, in which the aeroelastic airfoil exhibits chaotic motion, from viscous computational result is also vastly different from that for the inviscid solution as shown in Fig. 19. 


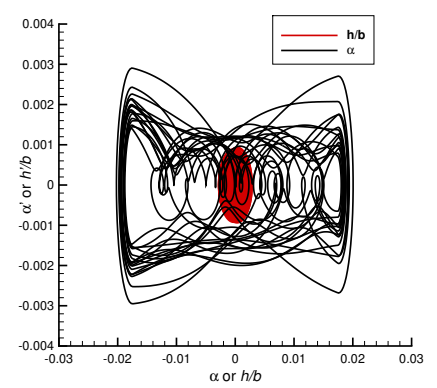

(a)

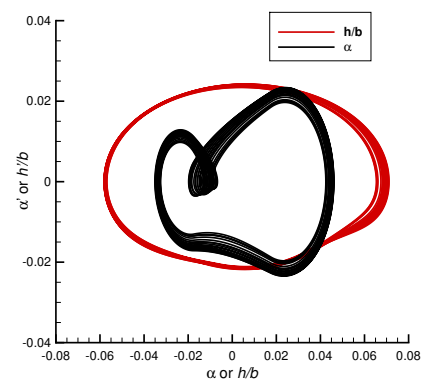

(c)

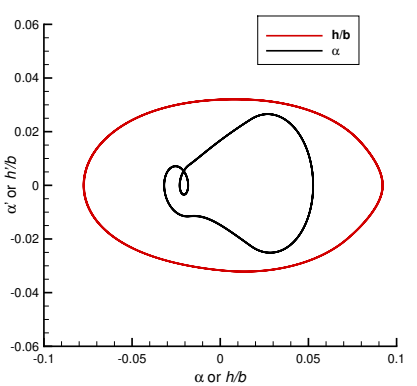

(e)

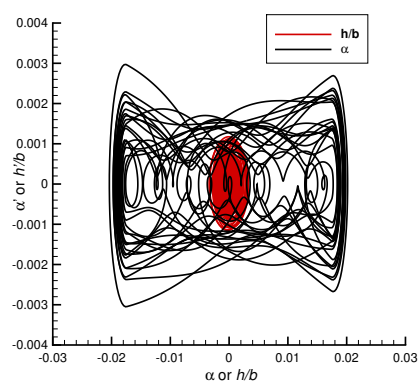

(b)

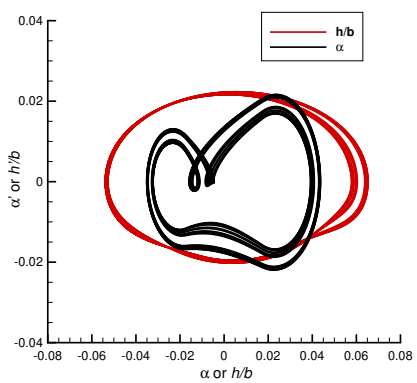

(d)

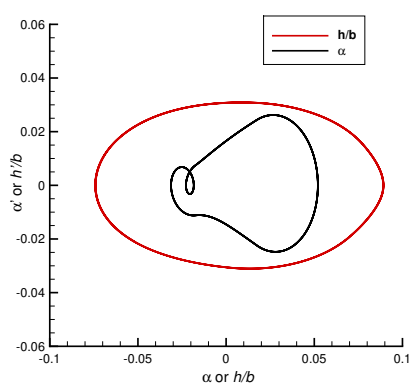

(f)

Figure 17: Phase plots of nonlinear aeroelastic system at Mach number 0.87 in inviscid air flow obtained by time marching approach based on: (a) CFD and (b) aerodynamic ROM at $U=0.4$, (c) CFD and (d) aerodynamic ROM at $U=1.455$, (e) CFD and (f) aerodynamic ROM at $U=1.678$ 


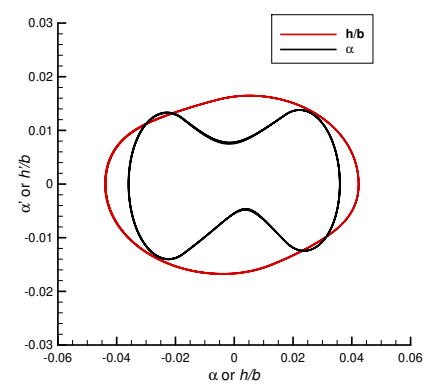

(a)

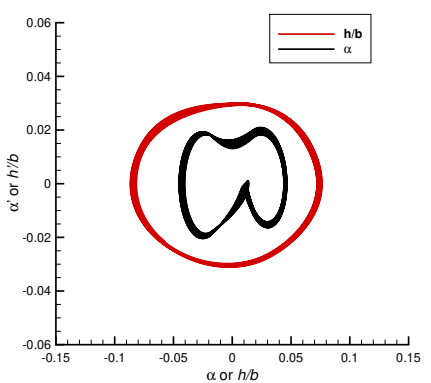

(c)

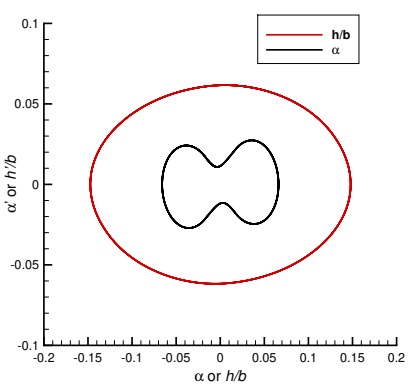

(e)

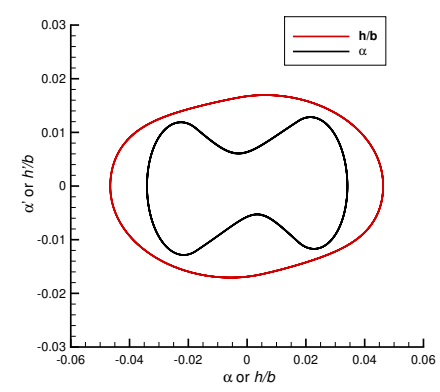

(b)

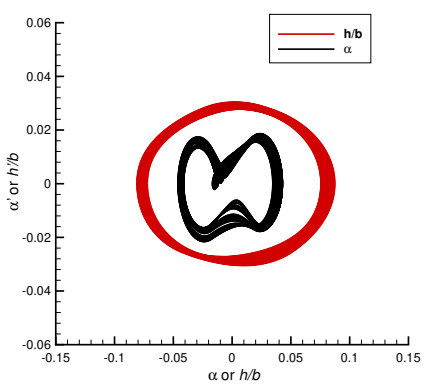

(d)

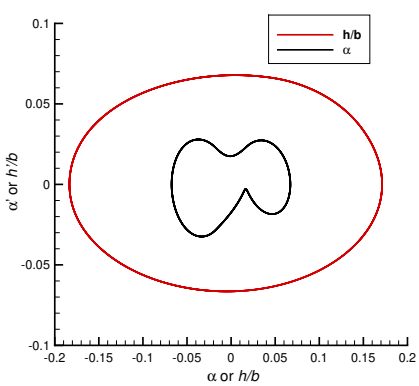

(f)

Figure 18: Phase plots of nonlinear aeroelastic system at Mach number 0.87 in viscous air flow obtained by time marching approach based on: (a) CFD and (b) aerodynamic ROM at $U / U_{l}=0.5128,(\mathrm{c}) \mathrm{CFD}$ and (d) aerodynamic ROM at $U / U_{l}=0.6$, (e) CFD and (f) aerodynamic ROM at $U / U_{l}=0.7692$. 


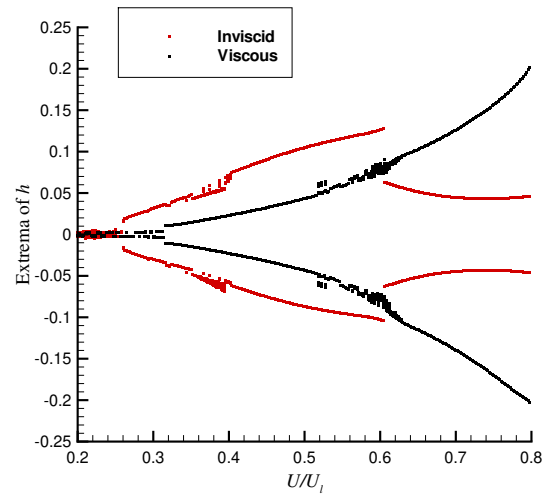

(a)

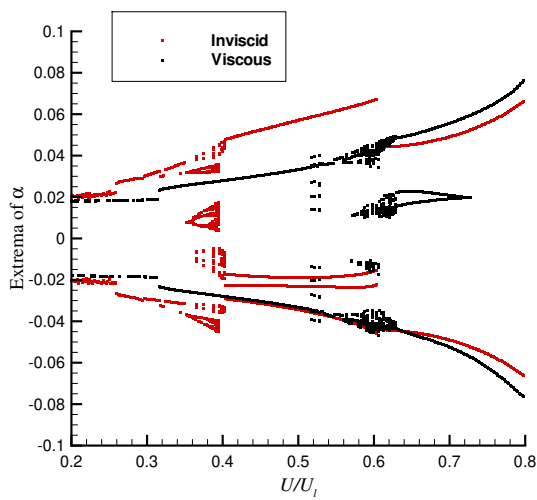

(b)

Figure 19: Comparison of bifurcation diagrams from inviscid and viscous solution at Mach number 0.87 obtained by aerodynamic ROM method: (a) plunging and (b) pitching.

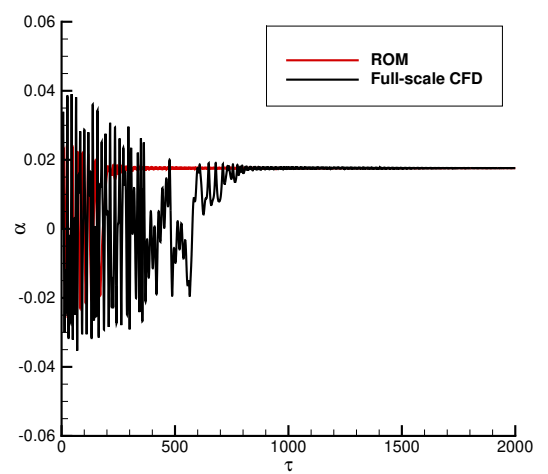

Figure 20: Nonlinear response at Mach number 0.87 at $U=0.4$ obtained by time marching approach based on Navier-Stokes equations. 


\subsection{Snap-through at different Mach numbers}

It has been noticed that the snap-through phenomenon observed at lowspeed in the inviscid flow may quench when aerodynamic viscosity is taken into account. It would be interesting to determine the Mach range in which the snap-through emerges for the viscous solution and the physical explanation of quenching of it in viscous flow. According to our previous study [16], the snap-through phenomena is substantially caused by the Single Degree of Freedom (SDOF) flutter and dual solution of SDOF aeroelastic system. So the key point should focus on the appearance of SDOF flutter in the following discussion.

According to Bendiksen [34, 35], with increasing Mach number, the Mach number freeze phenomenon happens when shock waves on the upper and lower surfaces reach the trailing edge of the airfoil. Meanwhile, the flow field around the airfoil becomes essentially independent of the freestream Mach number. It is easy to work out that the freeze Mach number for NACA $64 \mathrm{~A} 010$ is 0.91 according to our previous paper [6].

Smilg [36] noted that the SDOF flutter occurs in subsonic incompressible flow if the elastic axis of the airfoil is located at a point that is ahead of the airfoil quarter-chord (i.e. the aerodynamic center for thin airfoil in subsonic flow) but not too far ahead of the airfoil leading edge. Then Yang et al. [6] extend this conclusion to a transonic case based on numerous CFD calculations and flutter analysis. That is the SDOF flutter happens when the aerodynamic center of the airfoil lies behind the elastic axis, and it stops when the Mach number reaches the freeze Mach number.

Hence the relative locations of the aerodynamic center and elastic axis 


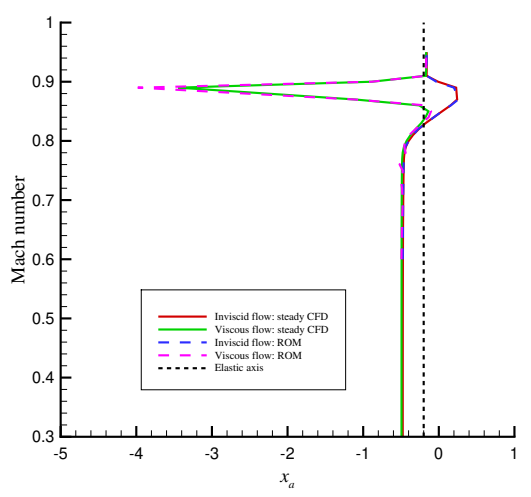

(a)

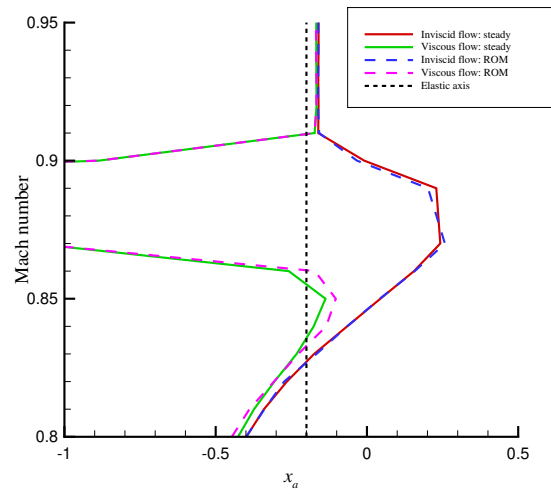

(b)

Figure 21: Aerodynamic center of NACA 64A010 at different Mach numbers: (a) overall, (b) detailed at transonic region.

at different Mach numbers should be examined first. According to the thin airfoil aerodynamic theory [17], the location of the aerodynamic center can be determined by

$$
x_{a c}=-2 c_{m \alpha} / c_{l \alpha}+a
$$

By using steady CFD analysis and the ROM method, we can obtain the slope of the aerodynamic forces coefficient at different Mach numbers and subsequently calculate the aerodynamic center of the airfoil. Fig. 21 presents the aerodynamic center of NACA 64A010 at different Mach numbers. Good agreement of the aerodynamic center from steady CFD and aerodynamic ROM is observed, demonstrating good accuracy of the ROM method again. It is evident that the aerodynamic center of the airfoil lies around the midpoint of the airfoil $\left(x_{a c}=-0.16\right)$ when the Mach number is greater than Mach 0.91, i.e. the freeze Mach number.

From the location of the aerodynamic center and the necessary condition 
for the appearance of SDOF flutter, we can infer that SDOF flutter can occur for $0.83<M a<0.9$ with inviscid aerodynamics for the present aeroelastic model. In the case of viscous flow, the aerodynamic center obtained from ROM method lies a little behind that from full-scale CFD as shown in Fig. 21 (b), the Mach number range for SDOF flutter obtained from these two approaches may differ slightly. SDOF flutter can happen for $0.84<M a<$ 0.86 from ROM method, which is wider than that of $0.84<M a<0.85$ predicted by steady CFD.

Stability analysis from ROM method is used to verify the Mach number range, where SDOF flutter occurs, predicted by the above condition. Only the pitching DOF of Eq.(18) is retained, and governing equation of SDOF aeroelastic system is obtained. From Yang et al. [6] and He et al. [37], it is known that only the aerodynamic model, rather than the structural parameters of the aeroelastic airfoil, determines the existence of SDOF flutter. Thus, the aeroelastic system with linear structural model is taken as the example to illustrate the Mach number at which SDOF flutter may occur. Fig. 22 shows the root locus of SDOF system at different Mach numbers. For the Euler results as displayed in Fig. 22 (a), SDOF flutter happens for $0.83<M a<0.9$, which agrees well with Mach number ranges satisfying the condition of SDOF flutter based on aerodynamic center. Together with Fig. 21 and Fig. 22 (b), it is easy to reach the same conclusion in the case of viscous flow.

To further verify the Mach number region of SDOF flutter and snapthrough phenomenon, the time marching approach with full-scale CFD simulation and aerodynamic ROM are performed on the original two-DOF aeroe- 


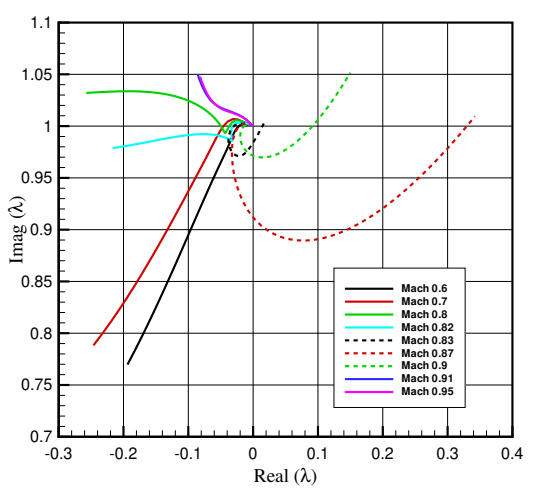

(a)

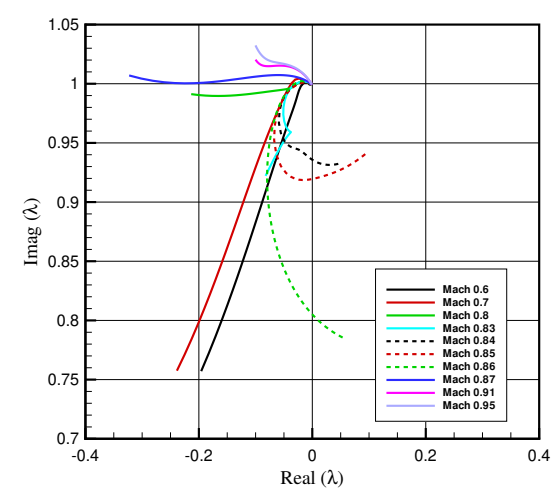

(b)

Figure 22: Root locus of single degree of freedom aeroelastic system with pitching DOF at different Mach numbers for : (a) inviscid solution, (b) viscous solution.

lastic system with free-play. As presented in Figs. 23 and 24, snap-through phenomena are observed at the low-speed region with different Mach numbers, which compares well the Mach regions predicted by the condition of SDOF flutter with respect to aerodynamic center and eigenvalue analysis of SDOF system.

So it can be seen that the location of the aerodynamic center plays an important role for SDOF flutter. The relative position between the aerodynamic center and elastic axis determines the appearance of SDOF flutter and snap-through before freeze Mach number. For the present NACA 64A010 airfoil, the inviscid-based aerodynamics can only provide a good prediction of the aerodynamic center for $M a<0.83$ and $M a>0.91$. However, the location of aerodynamic center obtained by Euler and Navier-Stokes solutions for $0.84<M a<0.9$ is significantly different. It leads to the varying behavior of the snap-through phenomenon for inviscid and viscous solutions. Thus, 


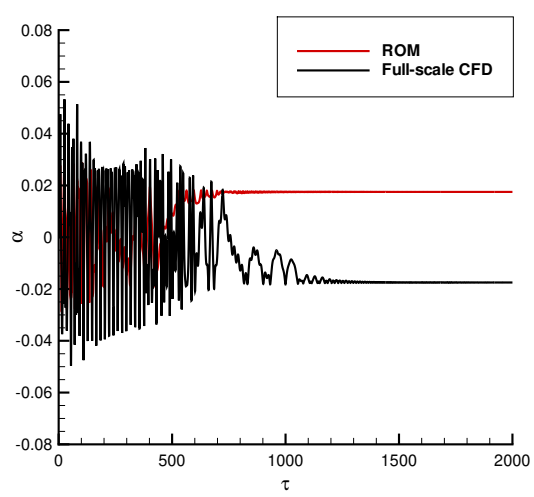

(a)

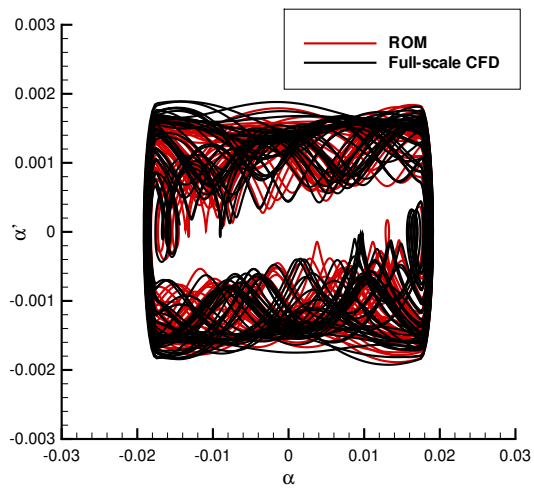

(c)

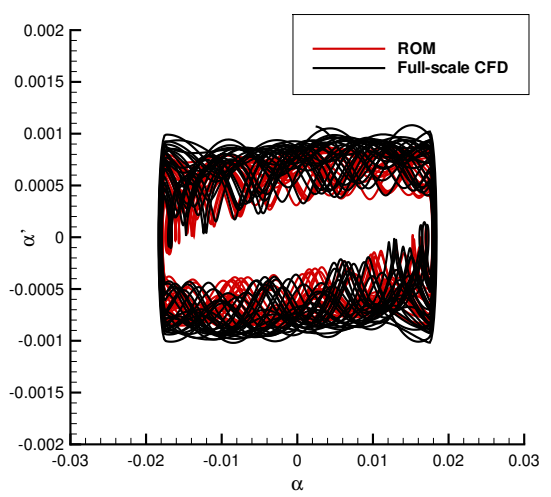

(b)

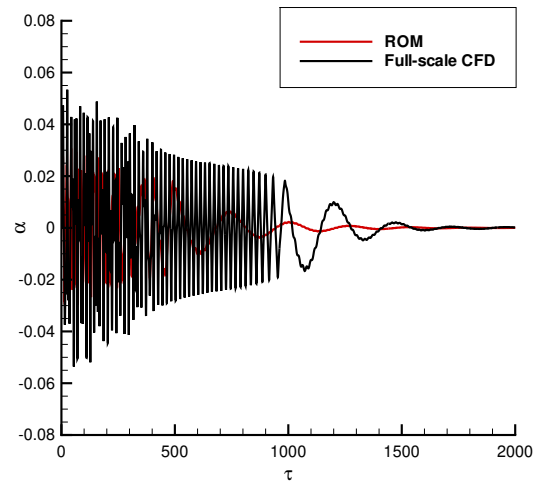

(d)

Figure 23: Aeroelastic response of pitching motion for inviscid solution at $U=0.4$ of: (a) Mach 0.82, (b) Mach 0.83, (c) Mach 0.9 and (d) Mach 0.91. 


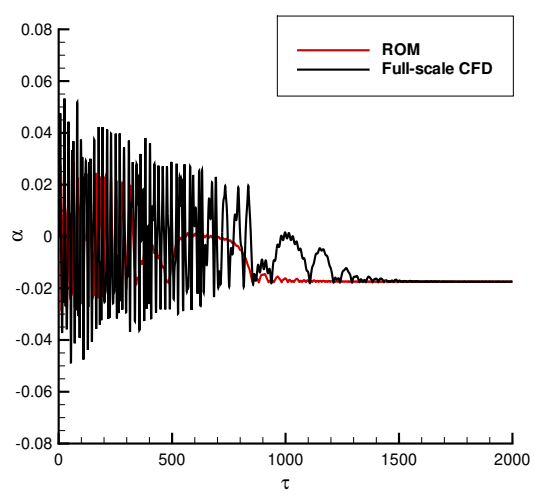

(a)

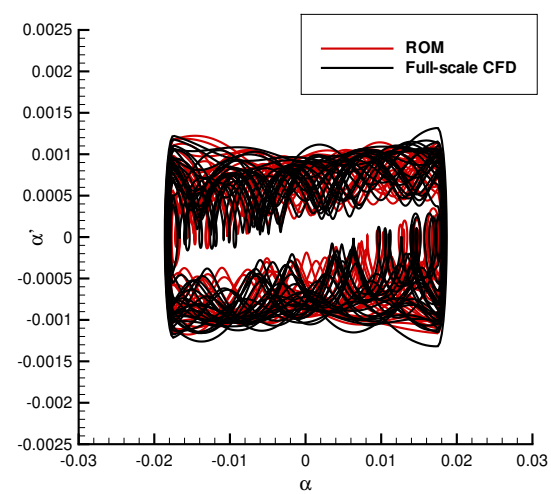

(c)

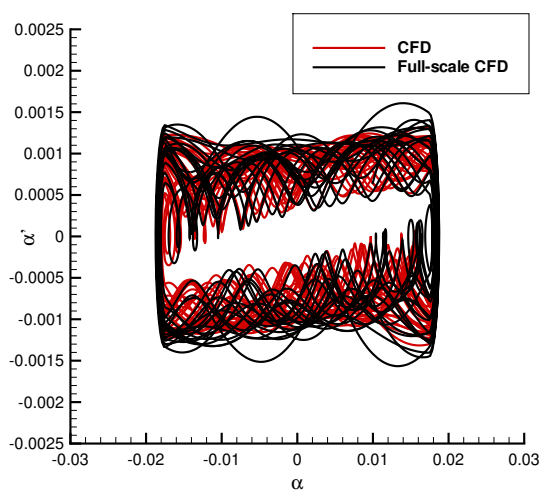

(b)

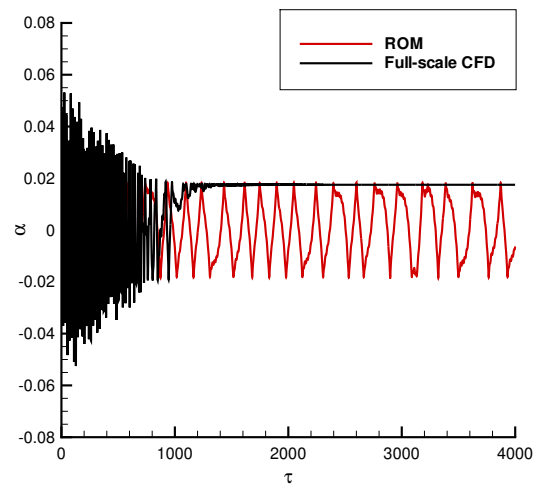

(d)

Figure 24: Aeroelastic response of pitching motion for viscous solution at: (a) $U=0.4$ of Mach 0.83, (b) $U=0.4$ of Mach 0.84, (c) $U=0.3$ of Mach 0.85 and (d) $U=0.4$ of Mach 0.86 . 


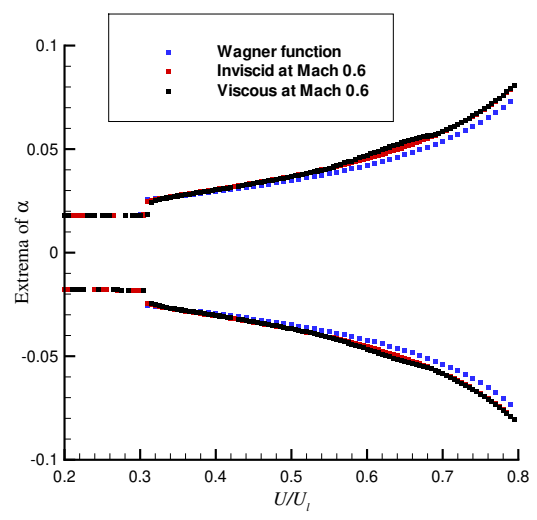

Figure 25: Comparison of bifurcation diagram from different methods in low subsonic flow.

the aerodynamic viscosity has a remarkable influence on the location of the aerodynamic center in the transonic regime and hence on the snap-through phenomenon.

\subsection{Bifurcations at different Mach numbers and complex nonlinear motions}

With the ROM built in the previous section, it is convenient to carry out the nonlinear aeroelastic response at different airspeeds at different Mach numbers. Firstly, the ROM method is verified by comparing the dynamic responses for aeroelastic airfoil at low Mach number with a conventional linear inviscid aerodynamic model. The Wagner's functions method is employed for comparison in the present study, and the bifurcation diagram for the aeroelastic model at Mach 0.6 in inviscid and viscous flow from ROM and at Mach 0 from Wager's function are presented in Fig. 25. Good agreements are observed indicating the feasibility of present methods, and it also demonstrates that only simple LCOs happen in low subsonic flow.

Figures 26 and 27 show the bifurcation diagrams at several typical Mach 
numbers for inviscid and viscous solutions, respectively. The nonlinear aeroelastic system experiences various type of bifurcations when the Mach number is changed. In the low Mach number region, regular subcritical bifurcations are observed, and only simple LCOs are observed. With increasing Mach number, complex LCOs and even non-periodic motions appear at specific airspeed regions. As the Mach number is increased further, regular subcritical bifurcations occur again, and only simple LCOs can be observed for different airspeeds. It is interesting to note that for both inviscid and viscous computational results, the complex dynamic response stops above Mach 0.91, which is the freeze Mach number. The Mach number range, at which complex nonlinear motion of the aeroelastic airfoil exists for inviscid aerodynamics, are quite different from that for viscous air flow.

A birth process of chaotic motion corresponding to Mach number for the Euler solution is observed. At low Mach number, conventional subcritical bifurcation happens. An additional sub-branch of the bifurcation diagram emerges at Mach 0.65, as shown in Fig. 26 (b). Since then the bifurcation diagram of the present aeroelastic system becomes more and more complex as Mach number increases, while the airspeed range in which chaotic motion takes place widens as well. Typical period-doubling bifurcations can be seen in the region of Mach 0.8 to 0.87; see Fig. 26 (d) and Fig. 19 (b). Then, the airspeed region with chaotic motion dies out gradually from Mach 0.88 to Mach 0.9 .

To overview the nonlinear dynamics behaviors based on Euler equations crossing the transonic region, phase plots of pitching DOF at different Mach number but at a fixed airspeed of $U / U_{l}=0.48$ are shown in Fig. 28. It 


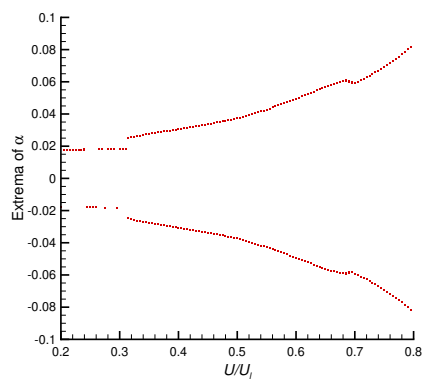

(a)

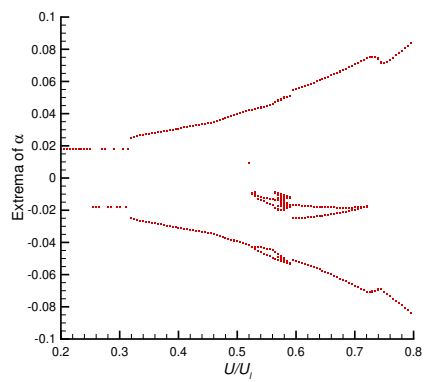

(c)

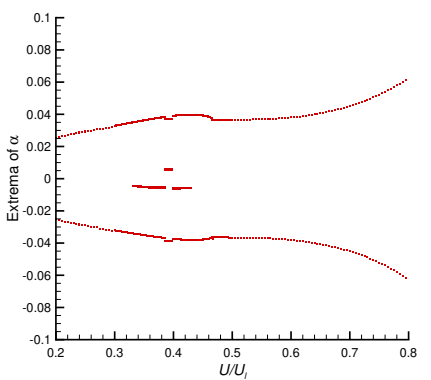

(e)

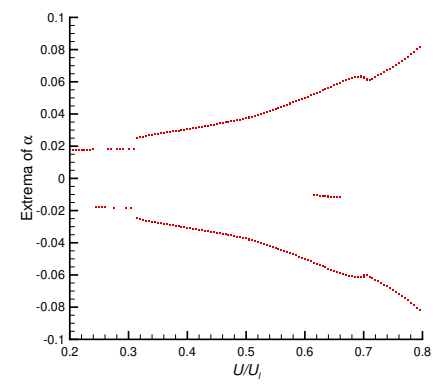

(b)

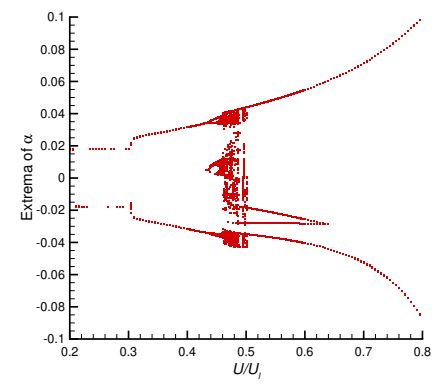

(d)

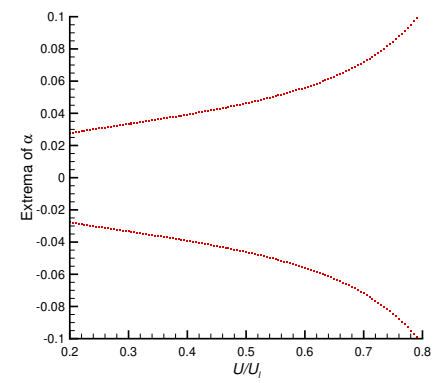

(f)

Figure 26: Bifurcation diagram obtained by aerodynamic ROM for inviscid solution: (a) Mach 0.64, (b) Mach 0.65, (c) Mach 0.7, (d) Mach 0.8, (e) Mach 0.9 and (f) Mach 0.91. 


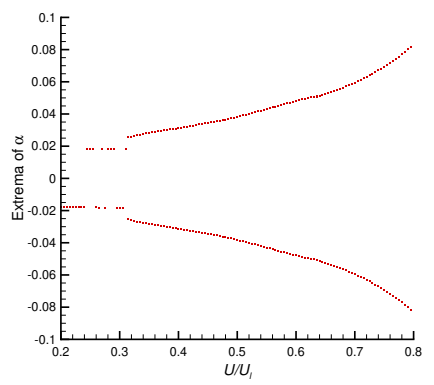

(a)

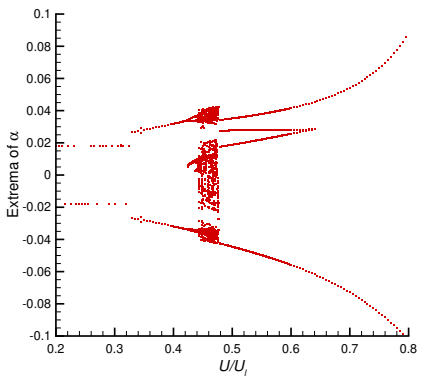

(c)

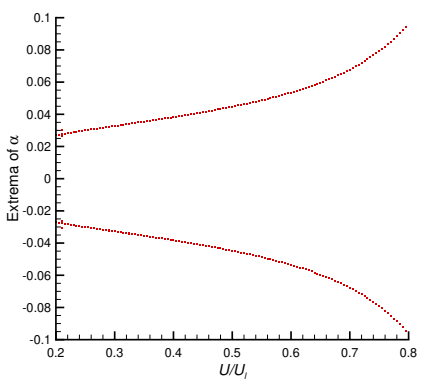

(e)

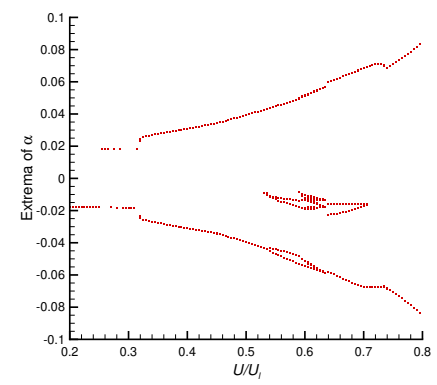

(b)

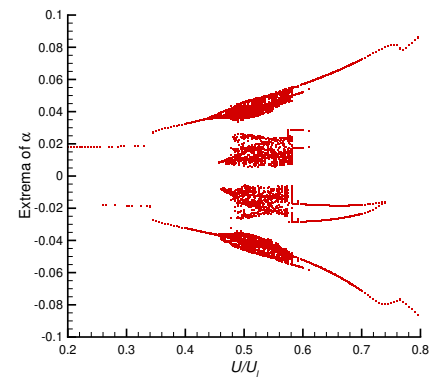

(d)

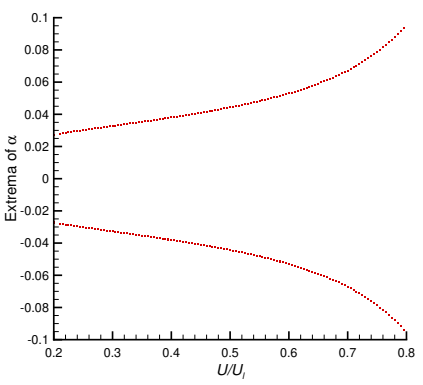

(f)

Figure 27: Bifurcation diagram obtained by aerodynamic ROM for viscous solution: (a) Mach 0.7, (b) Mach 0.71, (c) Mach 0.8, (d) Mach 0.9, (e) Mach 0.91 and (e) Mach 0.95. 
can be observed that at a low Mach number region (below Mach 0.66), the aeroelastic system oscillates in the same orbit as shown in Fig. 28 (a). With increasing Mach number from Mach 0.69 to 0.75, a birth and growth process of twists on phase portraits are observed in Fig. 28 (b). Subsequently, nonperiodic or chaotic motions are followed from Mach 0.78 to 0.8. A Poincaré map and power spectra for pitching motion at Mach 0.8, as a representation in chaos region, are used to identify chaotic motion, as shown in Fig. 29. Then the aeroelastic airfoil return to period-2 LCOs, Fig. 28 (e), and to simple LCO, Fig. 28 (f). It is interesting to note that for $M a>0.91$ as shown in Fig. 28 (f), the system responds with the same simple LCO, similar to the phenomenon in lower Mach number region. In summary, the present aeroelastic system experiences simple LCO, complex LCO, non-periodical oscillations, chaos, then back to complex LCO and simple LCO, when Mach number increases.

The trajectory of the aeroelastic system for the viscous solution is more complicated than that of the inviscid solution. In terms of various Mach number, the birth or death process of chaotic motion obtained by the NavierStokes equations is not as obvious as that from the Euler solution. The complex bifurcation come forth suddenly when the Mach number is larger than Mach 0.7, see Fig. 27 (a) and (b). Similarly, the chaotic region dies out within a small Mach number increment from Mach 0.9 to Mach 0.91 as shown in Fig. 27 (d) and (e).

Our detailed study in this section demonstrates that the Mach number of air flow is one important bifurcation parameter which can trigger perioddoubling bifurcation and chaotic motion of the aeroelastic system. The oc- 


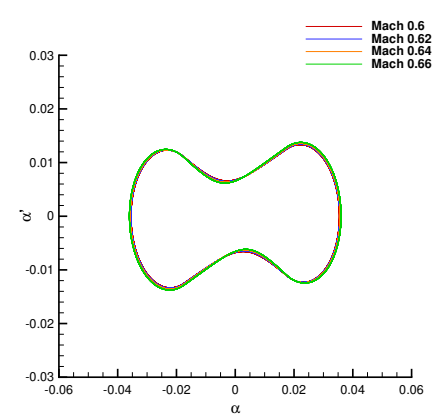

(a)

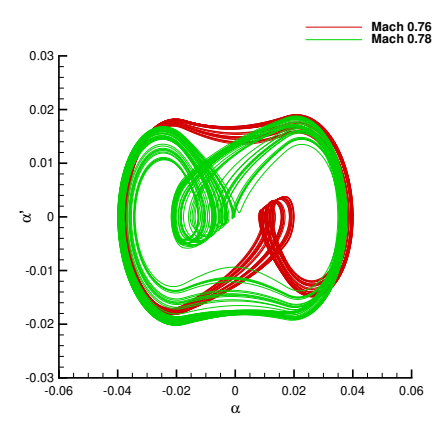

(c)

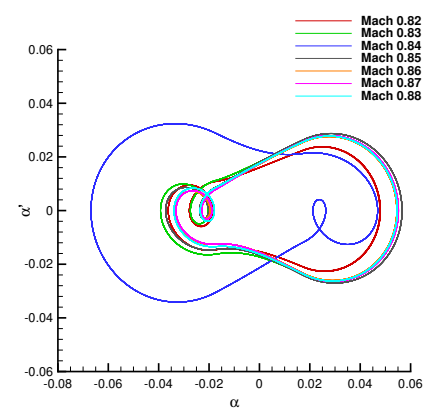

(e)

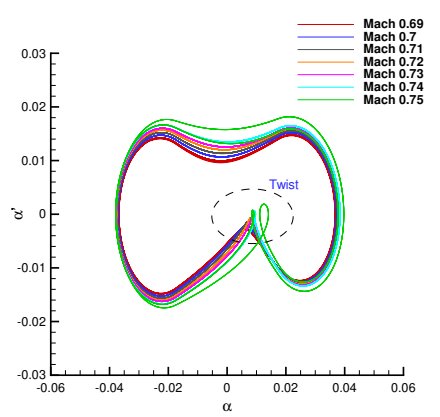

(b)

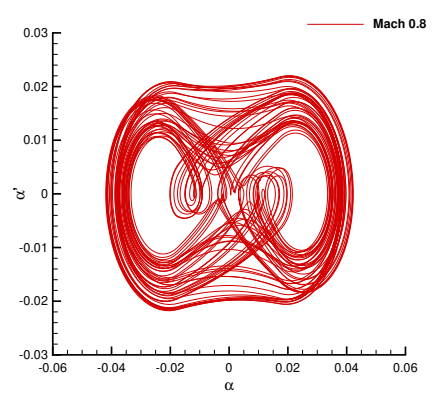

(d)

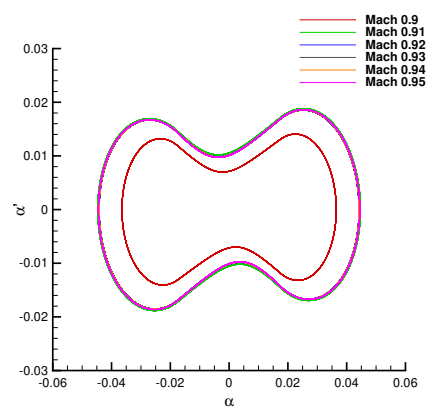

(f)

Figure 28: Phase plots obtained by aerodynamic ROM based on Euler equations at $U / U_{l}=$ 0.48 at: (a) $0.6<M a<0.66$, (b) $0.69<M a<0.75$, (c) $M a=0.76$ and 0.78 , (d) $M a=$ 0.8 , (e) $0.82<M a<0.88$ and (e) $0.9<M a<0.95$. 


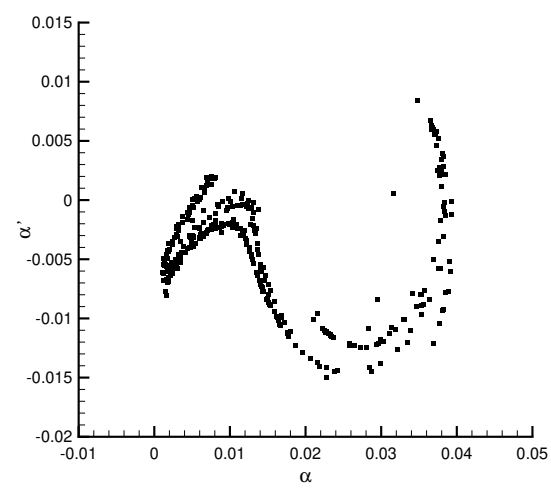

(a)

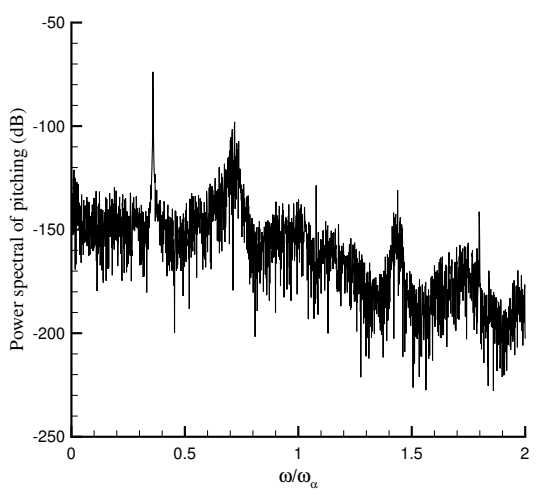

(b)

Figure 29: Nonlinear dynamics of pitching motion based on Euler equations at $U / U_{l}=0.48$ of Mach 0.8: (a) Poincaré map and (b) power spectra.

currence of complicated nonlinear aeroelastic phenomena tends to be much more common in transonic air flow than in subsonic and supersonic airflow.

In this section much effort is required to identify the influence of viscosity by comparing the aerodynamic influence coefficient derived from inviscid and viscous solutions. Since the frequency of nonlinear dynamic response usually distributes over a wide range, the AICs including $Q_{l h}, Q_{l \alpha}, Q_{m h}$ and $Q_{m \alpha}$ from 0.0 to 0.6 are compared and analyzed in the following sections.

Based on our numerous calculations, it is found that only simple LCOs happen for $M a<0.64$ and almost no difference of the bifurcation diagram is observed between the Euler and Navier-Stokes solutions at the same Mach number, see Fig. 25, Fig. 26 (a) and Fig. 27 (a). This is because AICs over this Mach number range, as shown in Fig. 30, are almost the same. Therefore, the viscosity has only minor importance in this scenario, and both inviscid and viscous aerodynamic models are suitable for predicting 
flutter solution and nonlinear dynamic behavior. From the point of view of aerodynamics, in this case the flow field around the airfoil is subsonic, and the effect of aerodynamic viscosity is limited and negligible, which agrees with the assumption of subsonic thin airfoil aerodynamic theory [17].

The most interesting scenario appears for $0.65<M a<0.82$, where neither strong shock nor severe flow separation exists around the airfoil. The inviscid-based model is capable of achieving linear flutter characteristics (see Fig. 13) and SDOF flutter solution (see Fig. 22). However, the bifurcation diagrams obtained from Euler-derived aerodynamics can be quite different from the Navier-Stokes solution. Taking Mach 0.7 as an example, a subcritical bifurcation is observed in viscous air flow and only simple LCOs are observed as shown in Fig. 27 (a), while the aeroelastic system undergoes a period doubling bifurcation for the inviscid solution as shown in 26 (c). We know that the nonlinear aeroelastic response is sensitive to the system parameter, so a small change of the variable, like the aerodynamic coefficients herein as shown in Fig. 31, can lead to a big difference of the nonlinear response. Thus, it may be sufficiently precise to carry out the linear flutter solution by using the inviscid aerodynamic model, while the viscous solution is still necessary to capture the nonlinear dynamic behavior in this Mach number range.

A significant effect of the aerodynamic viscosity can be observed for $0.83<M a<0.9$, in which strong shock wave and shock-boundary layer interaction occur in the flow field as shown in Fig. 32. Comparing with the pressure coefficient distribution shown in Fig. 32 (c), the inviscid solution tends to have a stronger shock that is close to the trailing edge. When 


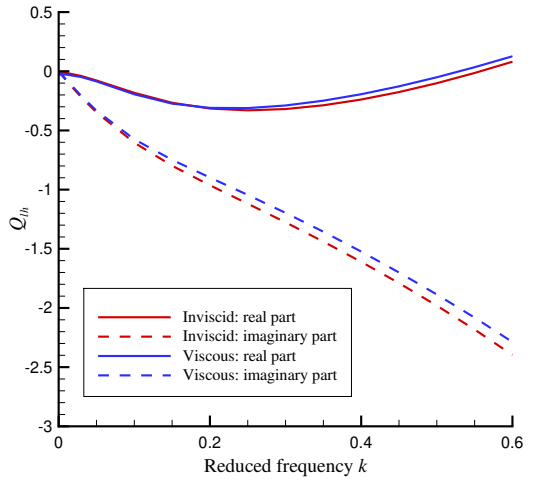

(a)

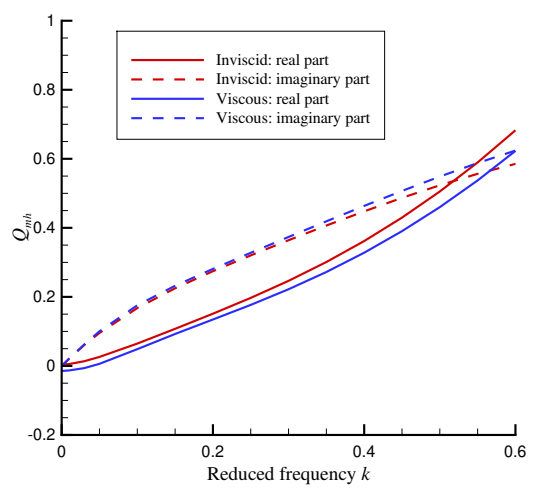

(c)

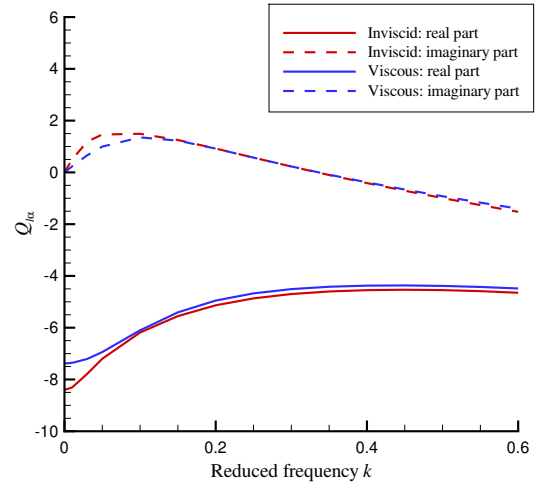

(b)

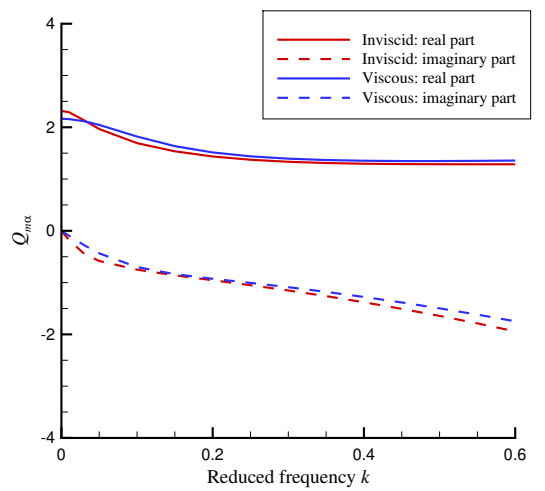

(d)

Figure 30: Comparison of aerodynamic influence coefficients at Mach 0.6 between inviscid and viscous solutions: (a) $Q_{l h}$, (b) $Q_{l \alpha}$, (c) $Q_{m h}$ and (d) $Q_{m \alpha}$. 


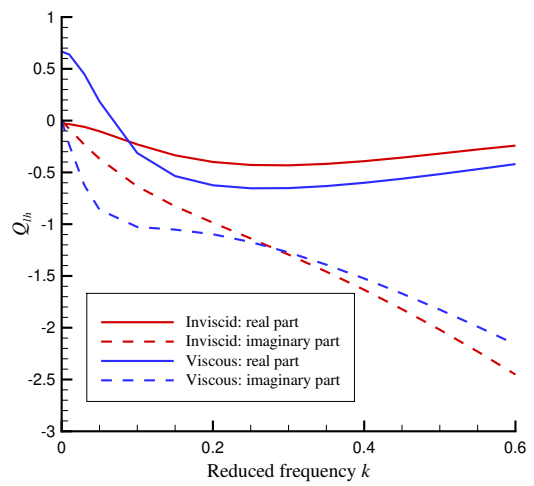

(a)

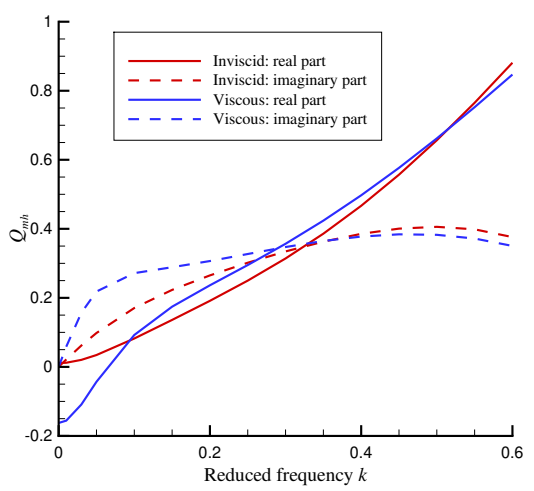

(c)

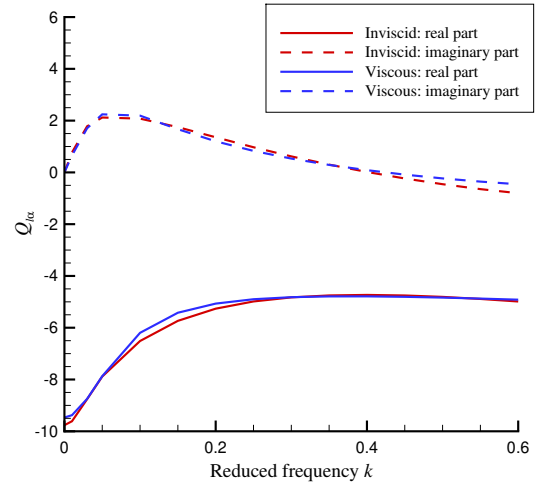

(b)

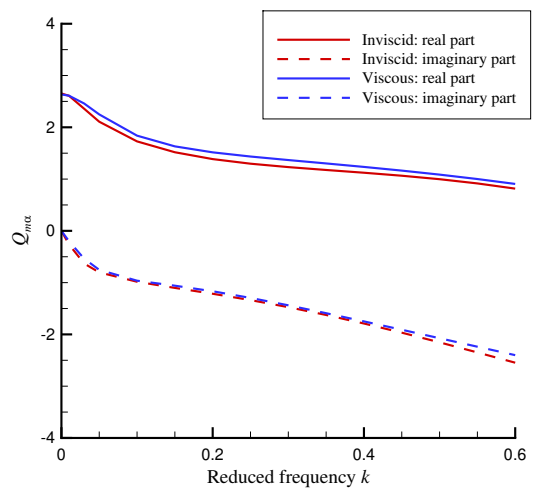

(d)

Figure 31: Comparison of aerodynamic influence coefficients at Mach 0.7 between inviscid and viscous solutions: (a) $Q_{l h}$, (b) $Q_{l \alpha}$, (c) $Q_{m h}$ and (d) $Q_{m \alpha}$. 
the viscosity is taken into account, the shock strength is reduced and the shock wave location is moved forward. As a result, the aerodynamic center from the inviscid aerodynamics is greatly different from the viscous solution as shown in Fig. 21. As to the unsteady characteristic of aerodynamics, a wide variation of the AICs between the inviscid and viscous solutions are observed in Fig. 33. Thus, both the shock wave and aerodynamic viscosity are important in this Mach number range. The viscous effect should be taken into consideration in the numerical analysis to obtain the linear flutter characteristic and nonlinear aeroelastic dynamic behavior.

It is interesting to find that when the Mach number is increased above 0.91, i.e. the freeze Mach number, no significant discrepancy of the bifurcation diagram is observed between the inviscid and viscous solutions. A slight difference of the AICs is observed as displayed in Fig. 35. At this moment, the shock wave becomes detached from the trailing edge as shown in Fig. 34 (a) and (b), and the pressure coefficient distribution are almost the same on the upper and lower surfaces of the airfoil as shown in Fig. 34 (c). It is obvious that the shock wave dominates the flow in this Mach number range and viscosity is of minor importance. So using the Euler equations is precise enough to capture the steady and dynamic behavior of the aerodynamics.

It should be noted that although three different aeroelastic phenomena are studied, namely the linear flutter characteristics, the snap-through phenomenon and the nonlinear dynamic behavior, the physical source of these are all the same. Different techniques, including root locus, SDOF flutter and AIC, are applied to identify the effect of viscosity on different type of aeroelastic phenomena and to elaborate how the viscosity impacts the aeroelastic 


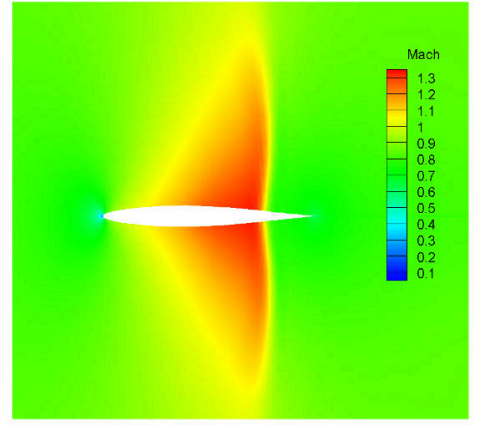

(a)

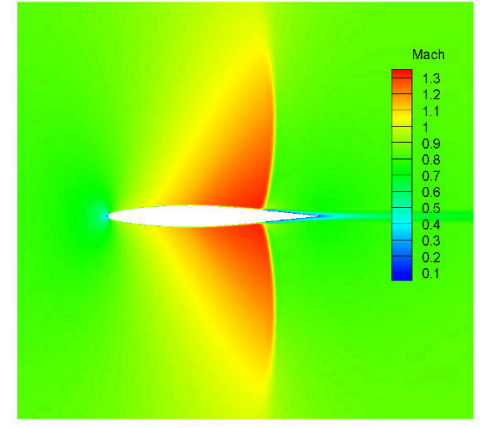

(b)

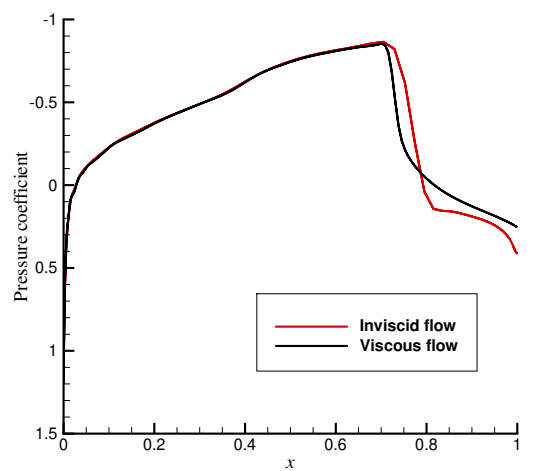

(c)

Figure 32: Flow field of NACA 64A010 at Mach 0.86: (a) Mach number distribution in inviscid air flow, (b) Mach number distribution in viscous air flow, (c) comparison of pressure distribution. 


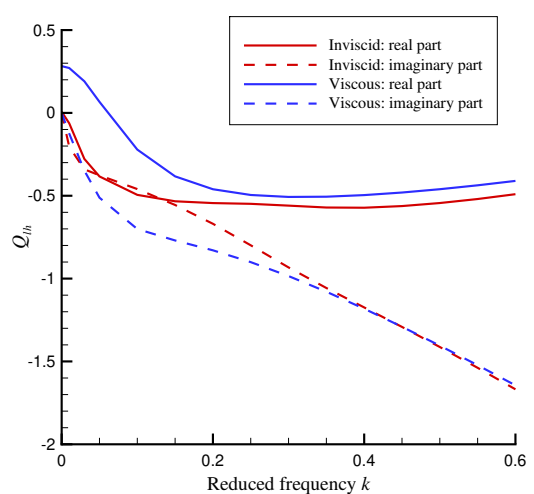

(a)

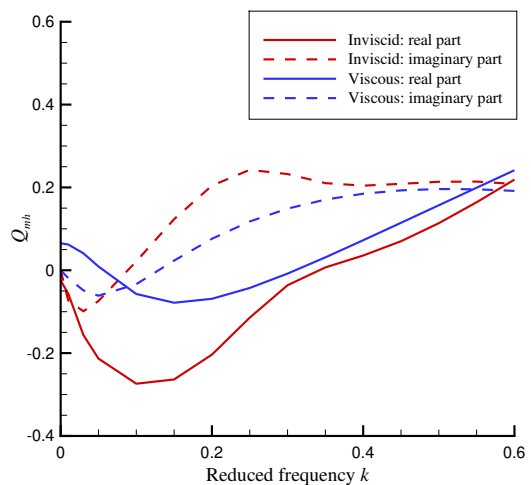

(c)

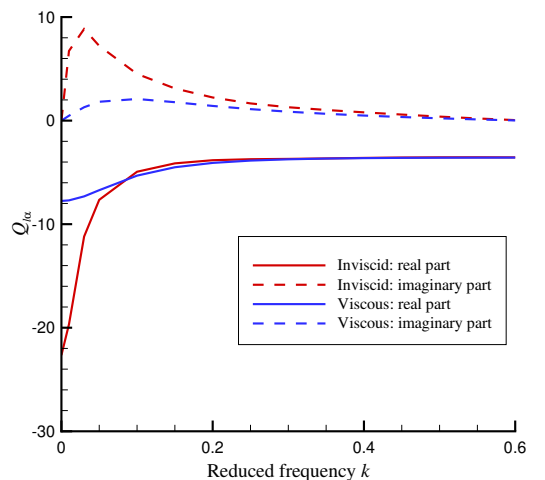

(b)

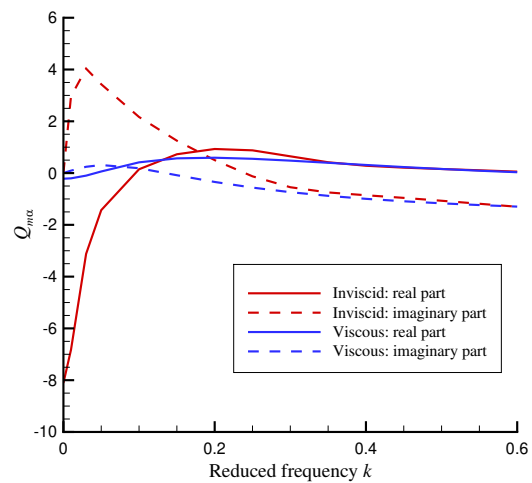

(d)

Figure 33: Comparison of aerodynamic influence coefficients at Mach 0.86 between inviscid and viscous solutions: (a) $Q_{l h}$, (b) $Q_{l \alpha}$, (c) $Q_{m h}$ and (d) $Q_{m \alpha}$. 


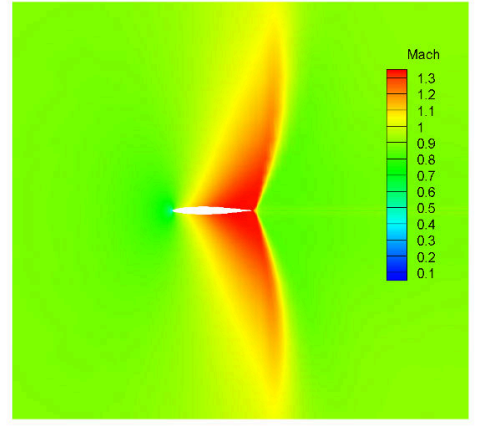

(a)

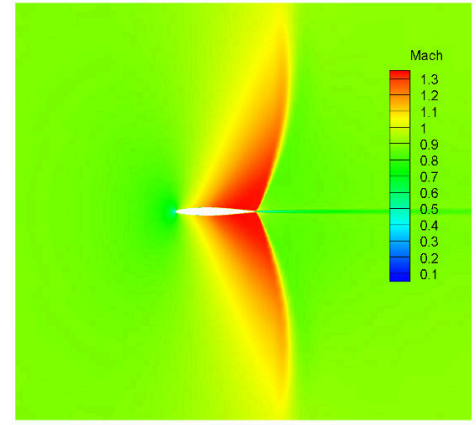

(b)

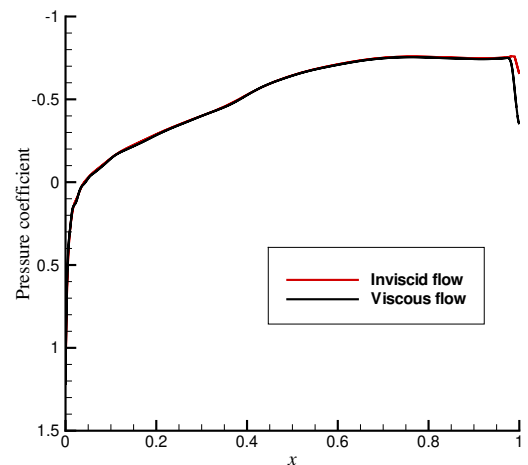

(c)

Figure 34: Flow field of NACA 64A010 at Mach 0.91: (a) Mach number distribution in inviscid air flow, (b) Mach number distribution in viscous air flow, (c) comparison of pressure distribution. 


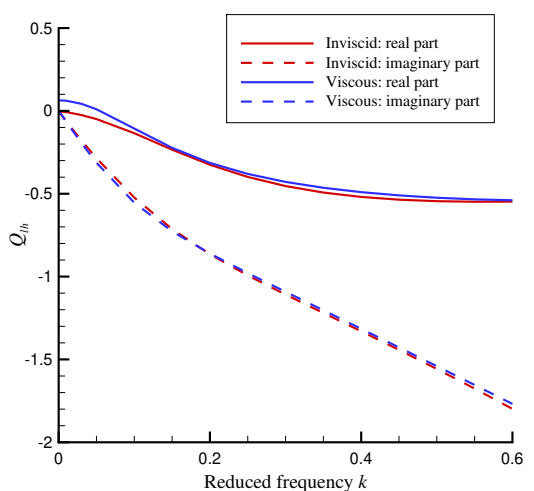

(a)

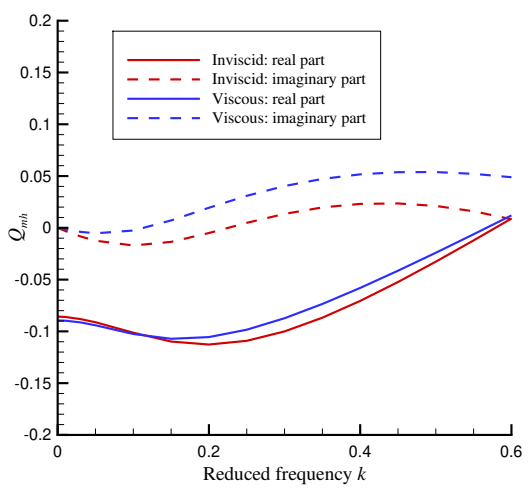

(c)

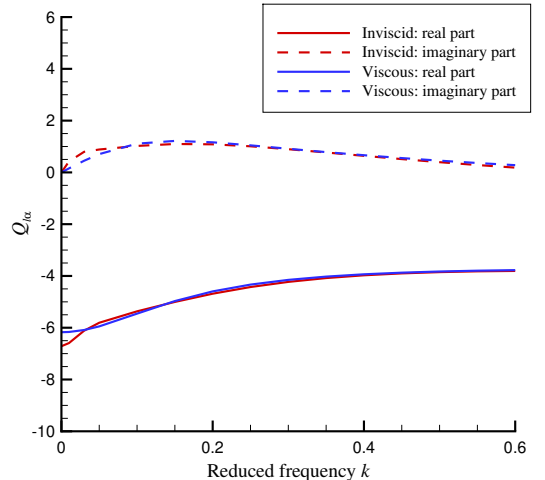

(b)

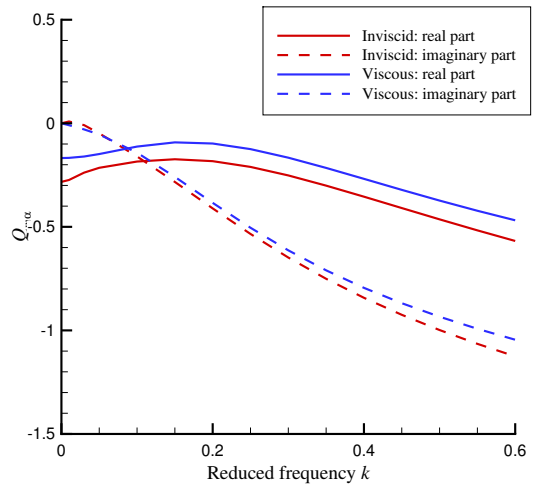

(d)

Figure 35: Comparison of aerodynamic influence coefficients at Mach 0.91 between inviscid and viscous solutions: (a) $Q_{l h}$, (b) $Q_{l \alpha}$, (c) $Q_{m h}$ and (d) $Q_{m \alpha}$. 
behaviors.

\subsection{The effect of damping on the nonlinear dynamic behavior}

To evaluate the effect of damping on the bifurcation behavior, the model at Mach 0.8 for inviscid solution is regarded as the baseline, in which a double-period bifurcation is observed as shown in Fig. 26 (d). The aerodynamic ROM method is implemented to obtain the nonlinear dynamic response considering damping due to its high efficiency. Fig. 36 displays the bifurcation diagrams of pitching motion for different damping coefficients. In the bifurcation diagram, a very large number of points occurs at a single speed, indicating that the nonlinear motion could be non-periodic or chaotic motion. With increasing damping coefficients $\zeta_{h}$ and $\zeta_{\alpha}$, the flutter speed increases as expected, while the speed region for complicated response reduces. There is no longer complex motions when the damping coefficients are sufficiently large as shown in Fig. 36 (b) and (c).

Next the effect of the damping on the snap-through phenomenon is examined. The nonlinear aeroelastic response at $U=0.3$ of Mach 0.85 for the viscous solution is taken as the baseline shown in Fig. 24 (c). Similarly, the ROM method is adopted to obtain the response with damping. Fig. 37 shows the phase plot from different damping coefficients, and it is observed that the damping of the structure can delay the snap-through to LCOs. When the damping is sufficiently large, the response becomes damped as shown in Fig. 37 (c). 


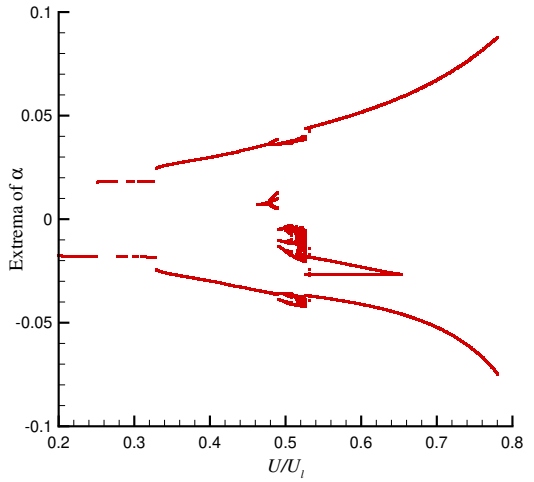

(a)

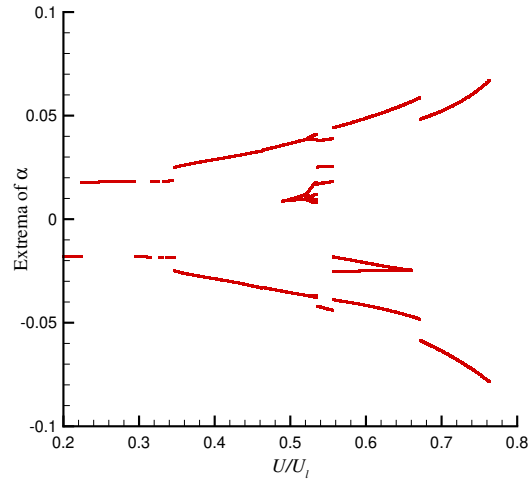

(b)

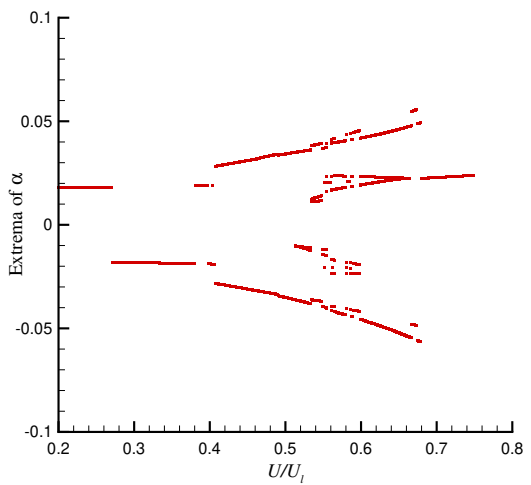

(c)

Figure 36: Effect of damping on the bifurcation diagram at Mach 0.8 based on Euler solution: (a) $\zeta_{h}=\zeta_{\alpha}=0.01$, (b) $\zeta_{h}=\zeta_{\alpha}=0.02$ and (c) $\zeta_{h}=\zeta_{\alpha}=0.03$. 


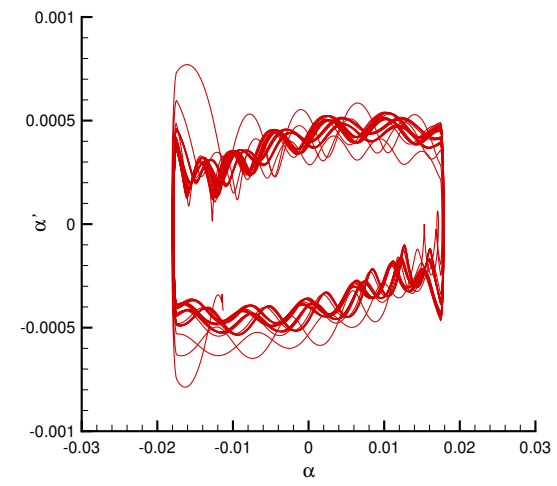

(a)

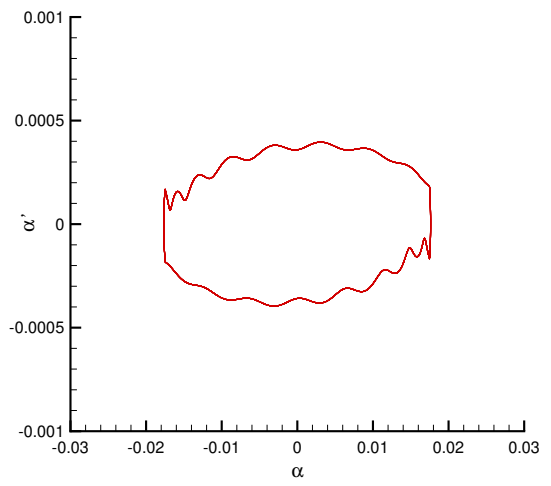

(b)

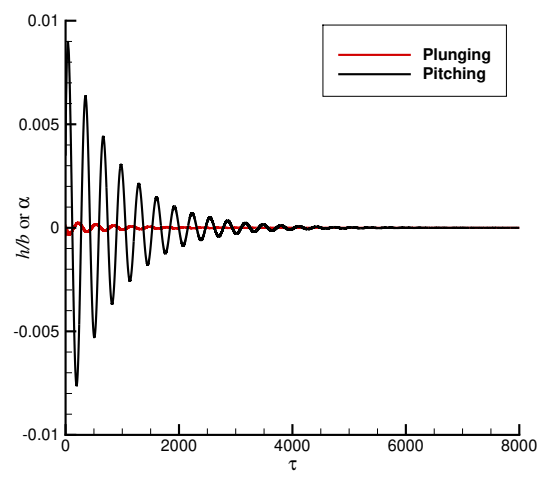

(c)

Figure 37: Effect of damping on snap-through phenomenon at $U=0.3$ of Mach 0.85 for viscous flow simulation: (a) $\zeta_{h}=\zeta_{\alpha}=0.01$, (b) $\zeta_{h}=\zeta_{\alpha}=0.02$ and (c) $\zeta_{h}=\zeta_{\alpha}=0.03$. 


\section{Conclusions}

Based on the Euler and Navier-Stoke equations, the nonlinear behavior of an aeroelastic airfoil with free-play in pitching DOF is studied in transonic air low. Fluent is implemented to carry out the transonic unsteady aerodynamic forces, and an RBF interpolation is applied to improve the capability of mesh deformation in Fluent via UDF. An aerodynamic interpolation technique and the Henon method are developed and applied in the time marching approach to obtain the nonlinear aeroelastic response. To reduce the computational cost, the ARX model is used to build an aerodynamic ROM for inviscid and viscous solutions, and a GA is employed to identify the model order.

The nonlinear aeroelastic system experiences various bifurcations as the Mach number increases. For low Mach numbers, regular subcritical bifurcations are observed. With increasing Mach number, complex LCOs and even non-periodic motions appear at specific airspeed regions. As the Mach number is increased higher than the freeze Mach number, regular subcritical bifurcations occur. Our detailed investigation demonstrates that Mach number is a vital bifurcation parameter which can trigger the period-doubling bifurcation and chaotic motion. Simulation in this paper shows that the complicated nonlinear aeroelastic phenomenon tends to occur easily in transonic air flow.

Comparisons with inviscid solutions are used to identify the effect of viscosity on the linear flutter, snap-through phenomenon and nonlinear dynamic behavior in transonic flow. The flow viscosity plays different roles at different Mach number ranges, which can be summarized as

- When Mach number is less than 0.64, the flow field around the airfoil is 
subsonic, and the effect of aerodynamic viscosity is limited and negligible. The viscosity has little effect on the aeroelastic phenomena. Both inviscid and viscous solution are suitable to simulate the aeroelastic response.

- When the flow is increased to $0.65<M a<0.82$, the effect of viscosity is multifarious on different type of aeroelastic phenomena. It has little effect on linear flutter characteristics, due to its limited influence on unsteady characteristics of the aerodynamics. But it has remarkable impact on the nonlinear dynamic behavior because of sensitivity to the system parameter in the nonlinear structure system. Thus, it is sufficiently precise to carry out the linear flutter solution by using the inviscid solution, while the viscous solution is still necessary to capture the nonlinear dynamic behavior in this Mach number range.

- The aerodynamic viscosity is significantly important for $0.83<M a<$ 0.9 , since strong shock wave and shock-boundary layer interaction occur in the flow field. It affects the unstable mechanism of flutter by shifting the unsteady aeroelastic mode. It greatly affects on the location of aerodynamic center and hence the snap-through phenomenon. In addition, the viscosity also plays an important role on the unsteady characteristic of aerodynamics, and also on the nonlinear aeroelastic response. Thus, the viscous effect should be considered to obtain the linear flutter characteristic and nonlinear aeroelastic dynamic behavior.

- When Mach number is above 0.91, the shock wave dominates the air flow and the viscosity is of minor importance. Use of Euler equations 
enables the capture of the linear flutter and nonlinear dynamic characteristics.

\section{Acknowledgments}

The authors acknowledge the financial support by Chinese SAFEA highend foreign expert program (GDW20163500194); National Natural Science Foundation of China (Grant No. 11472216, 11672240 and 11972079); Natural Science Basic Research Plan in Shaanxi Province of China (Program No. 2019JM-582). The first author would like to acknowledge Dr James Whidborne in Cranfield University for his comments and suggestions that improved this paper.

\section{References}

[1] K. Isogai, Numerical Study of Transonic Flutter of a Two-Dimensional Airfoil, TR-617T, National Aerospace Laboratory, Tokyo, Japan, 1980.

[2] J. P. Thomas, E. H. Dowell, K. C. Hall, Nonlinear Inviscid Aerodynamic Effects on Transonic Divergence, Flutter and Limit Cycle Oscillations, AIAA Journal 40 (2002) 638-646.

[3] Z. C. Yang, L. C. Zhao, Analysis of limit cycle flutter of an airfoil in incompressible flow, Journal of Sound and Vibration 123 (1988) 1-13.

[4] K. A. Kousen, O. O. Bendiksen, Limit Cycle Phenomena in Computational Transonic Aeroelasticity, Journal of Aircraft 31 (1994) 1257-1263. 
[5] D.-H. Kim, I. Lee, Transonic and Low-supersonic aeroealstic Analysis of a Two-degree-of-freedom Airfoil with a Freeplay Non-linearity, Journal of Sound and Vibration 234 (2000) 859-880.

[6] Z. Yang, S. He, Y. Gu, Transonic limit cycle oscillation behavior of an aeroelastic airfoil with free-play, Journal of Fluids and Structures 66 (2016) 1-18.

[7] A. Shishaeva, V. Vedeneev, A. Aksenov, G. Sushko, Transonic Panel Flutter in Accelerating or Decelerating Flow Conditions, AIAA Journal 56 (3) (2018) 997-1010.

[8] M. Candon, R. Carrese, H. Ogawa, P. Marzocca, C. Mouser, O. Levinski, W. A. Silva, Characterization of a 3DOF aeroelastic system with freeplay and aerodynamic nonlinearities-Part I: Higher-order spectra, Mechanical Systems and Signal Processing 118 (2019) 781-807.

[9] M. Candon, R. Carrese, H. Ogawa, P. Marzocca, C. Mouser, O. Levinski, Characterization of a 3DOF aeroelastic system with freeplay and aerodynamic nonlinearities-Part II: Hilbert-Huang transform, Mechanical Systems and Signal Processing 114 (2019) 628-643.

[10] E. H. Dowell, J. P. Thomas, K. C. Hall, Transonic Limit Cycle Oscillation Analysis Using Reduced Order Aerodynamic models, Journal of Fluids and Structures 19 (2004) 17-27.

[11] S. Munteanu, J. Rajadas, C. Nam, A. Chattopadhyay, Reduced-OrderModel Approach for Aeroelastic Analysis Involving Aerodynamic and Structural Nonlinearities, AIAA Journal 43 (2005) 560-571. 
[12] D. P. Jones, I. Roberts, A. L. Gaitonde, Identification of Limit Cycles for Picewise nonlinear aeroelastic systems, Journal of Fluids and Structures 23 (2007) 1012-1028.

[13] C. Gao, W. Zhang, Z. Ye, A new viewpoint on the mechanism of transonic single-degree-of-freedom flutter, Aerospace Science and Technology 52 (2016) 144-156.

[14] C. Gao, W. Zhang, X. Li, Y. Liu, J. Quan, Z. Ye, Y. Jiang, Mechanism of frequency lock-in in transonic buffeting flow, Journal of Fluid Mechanics 818 (2017) 528-561.

[15] C. Gao, W. Zhang, Z. Ye, Reduction of transonic buffet onset for a wing with activated elasticity, Aerospace Science and Technology 77 (2018) $670-676$.

[16] S. He, Z. Yang, Y. Gu, Nonlinear dynamics of an aeroelastic airfoil with free-play in transonic flow, Nonlinear Dynamics 87 (4) (2017) 2099-2125.

[17] J. D. Anderson Jr, Fundamentals of aerodynamics, Tata McGraw-Hill Education, 2010.

[18] S. Timme, K. Badcock, Transonic aeroelastic instability searches using sampling and aerodynamic model hierarchy, AIAA journal 49 (6) (2011) $1191-1201$.

[19] W. A. Silva, P. Chwalowski, B. Perry III, Evaluation of linear, inviscid, viscous, and reduced-order modelling aeroelastic solutions of the AGARD 445.6 wing using root locus analysis, International Journal of Computational Fluid Dynamics 28 (3-4) (2014) 122-139. 
[20] E. H. Dowell, R. Clark, D. Cox, J. H.C. Curtiss, J. W. Edwards, K. C. Hall, D. Peters, R. Scanlan, E. Simiu, F. Sisto, T. W. Strganac, A Modern Course in Aeroelasticity, Kluwer Academic Publishers, Dordrecht, 4th edn., 2004.

[21] A. De Boer, M. Van der Schoot, H. Bijl, Mesh deformation based on radial basis function interpolation, Computers \& structures 85 (11-14) (2007) 784-795.

[22] T. Rendall, C. Allen, Unified fluid-structure interpolation and mesh motion using radial basis functions, International Journal for Numerical Methods in Engineering 74 (10) (2008) 1519-1559.

[23] T. J. Cowan, A. S. Arena, K. K. Gupta, Accelerating computational fluid dynamics based aeroelastic predictions using system identification, Journal of Aircraft 38 (1) (2001) 81-87.

[24] W. Zhang, Z. Ye, Reduced-order-model-based flutter analysis at high angle of attack, Journal of Aircraft 44 (6) (2007) 2086-2089.

[25] MATLAB, version 9.2.0 (R2017a), The MathWorks Inc., Natick, Massachusetts, 2017.

[26] H. Dai, X. Yue, J. Yuan, D. Xie, S. Atluri, A comparison of classical Runge-Kutta and Henons methods for capturing chaos and chaotic transients in an aeroelastic system with freeplay nonlinearity, Nonlinear Dynamics 81 (1-2) (2015) 169-188.

[27] M. Henon, On the numerical computation of Poincaré maps, Physica D: Nonlinear Phenomena 5 (2) (1982) 412-414. 
[28] S. He, Z. Yang, Y. Gu, Transonic limit cycle oscillation analysis using aerodynamic describing functions and superposition principle, AIAA Journal 52 (7) (2014) 1393-1403.

[29] J. Alonso, A. Jameson, Fully-Implicit Time-Marching Aeroelastic Solutions, in: 32nd Aerospace Sciences Meeting, AIAA-94-0056, Reno, NV, 1994.

[30] B. Prananta, M. Hounjet, R. Zwaan, Two-dimensional transonic aeroelastic analysis using thin-layer Navier-Stokes method, Journal of Fluids and Structures 12 (6) (1998) 655-676.

[31] S. Yang, Z. Zhang, F. Liu, S. Luo, H.-M. Tsai, D. Schuster, Time-domain aeroelastic simulation by a coupled Euler and integral boundary-layer method, in: 22nd AIAA Applied Aerodynamic Conference, AIAA-20045377, Providence, RI, 2004.

[32] K. J. Badcock, S. Timme, S. Marques, H. Khodaparast, M. Prandina, J. Mottershead, A. Swift, A. Da Ronch, M. Woodgate, Transonic aeroelastic simulation for instability searches and uncertainty analysis, Progress in Aerospace Sciences 47 (5) (2011) 392-423.

[33] R. M. Bennett, R. N. Desmarais, Curve fitting of aeroelastic transient response data with exponential functions, in: Flutter Testing Techniques, NASA SP-415, NASA, Washington, D. C., 43-58, 1976.

[34] O. Bendiksen, Transonic stabilization laws for unsteady aerodynamics and flutter, in: 53rd AIAA/ASME/ASCE/AHS/ASC Structures, Structural Dynamics and Materials Conference, 1718, 2012. 
[35] O. O. Bendiksen, Transonic flutter characteristics of advanced fighter wings, in: 56th AIAA/ASME/ASCE/AHS/ASC Structures, Structural Dynamics, and Materials Conference, AIAA-2015-0438, Kissimmee, FL, 2015.

[36] B. Smilg, The instability of pitching oscillations of an airfoil in subsonic incompressible potential flow, Journal of the Aeronautical Sciences 16 (11) (1949) 691-696.

[37] S. He, Z. Yang, Y. Gu, Limit cycle oscillation behavior of transonic control surface buzz considering free-play nonlinearity, Journal of Fluids and Structures 61 (2016) 431-449. 
2019-12-16

Nonlinear aeroelastic behavior of an airfoil with free-play in transonic flow

He, Shun

Elsevier

He S, Guo S, Li W, et al., (2019) Nonlinear aeroelastic behavior of an airfoil with free-play in transonic flow. Mechanical Systems and Signal Processing, Volume 138, April 2020, Article number 106539

https://doi.org/10.1016/j.ymssp.2019.106539

Downloaded from Cranfield Library Services E-Repository 